\title{
High-fidelity trajectory design to flyby near-Earth asteroids using $\mathrm{Cu}-$ beSats
}

\author{
P. Machuca ${ }^{\mathrm{a}, *}$, J. P. Sánchez ${ }^{\mathrm{a}}$, J. J. Masdemont ${ }^{\mathrm{b}}$, G. Gómez ${ }^{\mathrm{c}}$
}

a Space Research Group, Cranfield University, Cranfield, United Kingdom

${ }^{\mathrm{b}}$ IEEC \& Departament de Matemàtiques, Universitat Politècnica de Catalunya, Barcelona, Spain

${ }^{c}$ IEEC \& Departament de Matemàtiques i Informàtica, Universitat de Barcelona, Barcelona, Spain

\begin{abstract} the impulsive results $( \pm 15 \mathrm{~m} / \mathrm{s})$.

Keywords:

Trajectory design

Interplanetary CubeSats

Near-Earth asteroids

Low-thrust trajectories

Sun-Earth Lagrange points
\end{abstract}

Fast development of CubeSat technology now enables the first interplanetary missions. The potential application of CubeSats to flyby near-Earth asteroids is explored in this paper in consideration of CubeSats' limited propulsive capabilities and systems constraints. Low-energy asteroid flyby trajectories are designed assuming a CubeSat is initially parked around to the Sun-Earth Lagrange points. High-impulse and low-thrust trajectories with realistic thrusting models are computed first in the Circular Restricted ThreeBody Problem (CR3BP), and then in a high-fidelity ephemeris model. Analysis in the ephemeris model is used to confirm that trajectories computed in the CR3BP model also exist in a more realistic dynamical model, and to verify the validity of the results obtained in CR3BP analysis. A catalogue of asteroid flyby opportunities between years 2019 and 2030 is provided, with $80 \mathrm{~m} / \mathrm{s}$ of available $\Delta \mathrm{V}$ and departure from halo orbits around the first and second Sun-Earth Lagrange points (of similar size to those typically used by scientific missions). Results show that the CR3BP model can serve as an effective tool to identify reachable asteroids and can provide an initial estimation of the $\Delta \mathrm{V}$ cost in the ephemeris model (with $\pm 15 \mathrm{~m} / \mathrm{s}$ accuracy). An impulsive maneuver model can also provide an accurate estimation of the $\Delta \mathrm{V}$ requirement for a CubeSat equipped with a high-impulse thruster (with $4 \mathrm{~m} / \mathrm{s}$ accuracy), even if its thrust magnitude is small and requires duty cycling; low-thrust $\Delta \mathrm{V}$ requirements, however, may differ significantly from

Nomenclature

Acronyms and abbreviations

au

CR3BP

EMB

EoM

GA

JWST

LEO

LSST

L1, L2

NEO

NLP

PUC

SLS
Astronomical unit

Circular Restricted Three-Body Problem

Earth-Moon barycenter

Equations of motion

Genetic algorithm

James Webb Space Telescope

Low-Earth orbit

Large Synoptic Survey Telescope

First and second Sun-Earth barycentric Lagrange points

Near-Earth object

Non-linear programming

Propulsion Unit for CubeSats

Space Launch System
STM

TOF

$\mathrm{U}$

Symbols

$\underline{\boldsymbol{A}}$

$\beta$

$d, r$

$\delta($.

$\Delta($.

$\Delta t_{\boldsymbol{T}_{1}}, \Delta t_{\boldsymbol{T}_{2}}$

$\Delta v_{1}, \Delta v_{2}$

$\Delta \mathrm{V}$
State-transition matrix

Time of flight

CubeSat basic unit (10 cm x 10 $\mathrm{cm} \times 10 \mathrm{~cm})$

Jacobian matrix of the equations of motion

Normalized $z$-amplitude of halo orbit

Non-dimensional distances to Sun and EMB, respectively

Variable variation

Variable difference

Duration of first and second thrust arcs, respectively

First and second impulsive maneuvers, respectively

Velocity increment

* Corresponding author.

E-mail address: p.machuca@ cranfield.ac.uk (P. Machuca). 


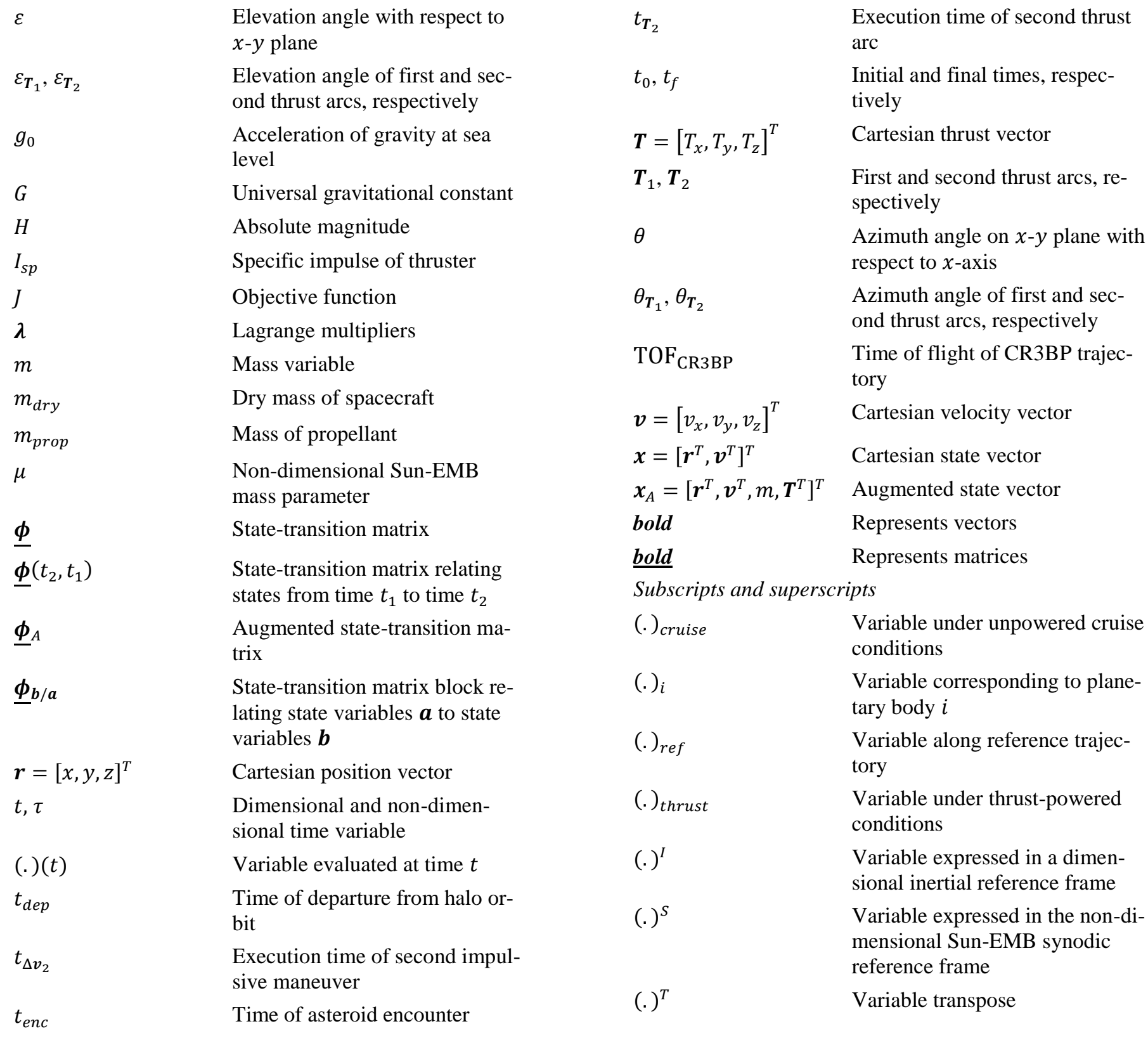

\section{Introduction}

CubeSat capabilities have rapidly improved in recent years. Due to their lower development and implementation costs compared to traditional space missions, CubeSat technology is seen as a potential solution for the low-cost exploration of the Solar System. In 2018, the first CubeSat mission beyond LEO was launched, MarCO, along NASA's InSight mission to flyby Mars [1,2]. Several other interplanetary CubeSat missions are planned for the early 2020s to explore cislunar space, observe and land on the Moon, and flyby asteroids [3,4].

The potential application of CubeSat technology for planetary exploration is today an active field of research. Provided their limited propulsive capability, interplanetary CubeSat missions generally leverage a piggyback opportunity to reach their destinations beyond LEO. Relevant mission examples include those of the 6U CubeSats to explore cislunar space, the Moon, and a near-Earth asteroid as secondary payloads to NASA's SLS [3], 6U CubeSats to complement the scientific objectives of ESA's Hera mission at the Didymos system [4], or the <10-kg asteroid landers that piggybacked on board JAXA's Hayabusa-2 mission to Ryugu [5]. 
This paper provides further analysis on the design of trajectories to study near-Earth asteroids under the constraints of a CubeSat platform. Near-Earth asteroid reachability studies within a stringent $\Delta \mathrm{V}$ budget can be found in previous literature, with several noteworthy examples. Greco et al. [6] computed rendezvous and flyby trajectories with an Earth departure for a low-thrust 12U CubeSat mission between years 2024 and 2034. Flyby missions of less than 1000 days are found possible with $\Delta \mathrm{V}$ budgets as low as $30 \mathrm{~m} / \mathrm{s}$, and with $600 \mathrm{~m} / \mathrm{s}$ for a rendezvous mission. Strange et al. [7] computed rendezvous and sample-return requirements from Earth-escape trajectories using chemical and low-thrust propulsion between years 2020 and 2024. Results show that $150 \mathrm{~m} / \mathrm{s}$ of $\Delta \mathrm{V}$ with a Mars or Venus gravity-assist maneuver allow rendezvous missions of less than 5 years and sample-return missions of less than 8 years. Campagnola et al. [8] designed the low-thrust flyby trajectory for the 50-kg PROCYON mission, leveraging an Earth gravity assist one year after deployment, and $150 \mathrm{~m} / \mathrm{s}$ of $\Delta \mathrm{V}$. Finally, Pezent et al. [9] designed rendezvous and low-speed flyby trajectories for the 6U NEA Scout CubeSat mission, exploiting a Moon flyby, trajectories of up to 3 years in duration, and a solar sail propulsion system.

Previous work found in literature generally leverages either propulsion capabilities of hundreds of meters per second, gravity-assist maneuvers around the Earth, Moon or other planets (which add complexity to the mission), or trajectories of several years to reach their targets. This paper presents a methodology to compute asteroid flyby trajectories in consideration of CubeSat-specific mission constraints, such as a simple mission profile, very low total $\Delta \mathrm{V}$ (below $80 \mathrm{~m} / \mathrm{s}$ ), heavy duty cycling, low thrust magnitudes, and short transfer times to the asteroids (below 150 days). In view of the modest overall performance of CubeSats (e.g., communications, power, life expectancy, environmental constraints, etc.), a short-duration flyby trajectory is considered to enforce a realistic demand on the spacecraft subsystems: for instance, to avoid far communication distances from Earth and to limit the time over which trajectory uncertainties are propagated (which ultimately drive the accuracy and altitude of the asteroid flybys).

The mission concept here considered assumes a CubeSat is initially parked in an orbit around the first or second SunEarth barycentric Lagrange points, from where it can then perform its own propulsive maneuvers and flyby a near-Earth asteroid. Several large scientific missions to L1 and L2 are planned for the next decade [10-15], and various launch scenarios could be envisioned for the CubeSat mission. As examples, a dedicated launch or a larger scientific mission could deploy a CubeSat directly into an orbit around L1 or L2, or a CubeSat could be deployed into a free-insertion transfer trajectory to the Lagrange points. This kind of transfer trajectories have and will be used by several missions such as Herschel/Planck, Euclid, ARIEL or PLATO [13-16], as they require only minimal correction maneuvers to reach the Lagrange points: to remove launcher dispersions and correct perigee velocity. Once in an orbit around L1 or L2, the natural dynamics around these points enable asteroid flyby trajectories with very low $\Delta \mathrm{V}$ requirements [17,18], and thus present an interesting opportunity for an asteroid exploration mission using CubeSats.

Previous work by Machuca et al. [18] considered the same mission concept but contained only a preliminary analysis for a semi-autonomous CubeSat mission to an asteroid: a systems design was proposed along with a detailed discussion on CubeSat system implications, impulsive flyby trajectories between years 2019 and 2025 were computed only in the medium-fidelity CR3BP, flyby accuracies through autonomous navigation were assessed for reduced ground operations costs, and the potential scientific return of the mission was discussed. From this preliminary analysis, it is also clear that constraining the transfer time to the asteroid - and therefore limiting the propagation of uncertainties - is beneficial for the resulting flyby accuracies, given the limited estimation accuracies that can be obtained solely through autonomous navigation.

Analysis in [18] already identified several challenges to the mission concept, such as large flyby velocities or constraining allocation and performance of sensors, actuators, and science payload. As such, the science return was foreseen to be modest and limited to medium-resolution images and spectroscopic observations of the asteroid, which could, for instance, support taxonomic studies and help calibrate and validate ground-based observations. Although modest science is anticipated, the mission concept provides a simple mission architecture, with very low $\Delta \mathrm{V}$ requirements, with low development and implementation costs, and with the ability to incorporate high levels of autonomy that can achieve significant science and demonstrate technology at an orders-of-magnitude lower cost than traditional missions. Furthermore, the mission concept is certainly scalable from CubeSats to SmallSats and to several-hundred-kilogram spacecraft that could leverage the same kind of launch opportunity.

In this paper, and as an extension of the work in [18], not only impulsive trajectories but also low-thrust and noninstantaneous high-impulse trajectories are computed for the 2019-2030 time window. These flyby trajectories are first computed in the CR3BP considering the gravitational influences of the Sun and the Earth-Moon barycenter only, as in [18], and then their existence in a higher-fidelity dynamical model is verified (i.e., ephemeris model), considering the 
actual positions and gravitational attractions of the major influencing bodies in the Solar System. In addition, a realistic thrusting model is implemented in which non-instantaneous maneuvers and duty cycling are considered. The small thrust magnitudes that can be generated by current CubeSat propulsion systems, along with heavy duty-cycling strategies for proper thermal control of the thruster, result in non-impulsive propulsive maneuvers of long duration compared to those of traditional spacecraft. Thus, analysis of the effect of the non-instantaneity of propulsive maneuvers in CubeSat missions is also included in this paper.

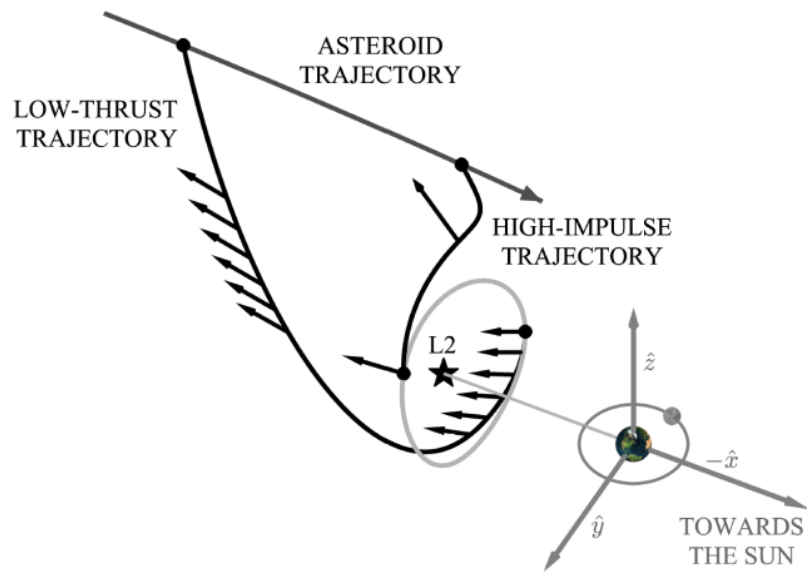

Fig. 1. High-impulse and low-thrust flyby trajectories from Sun-EMB L2 halo orbit (in Sun-EMB synodic reference frame).

This work therefore identifies asteroid flyby opportunities for a CubeSat (or SmallSat) mission under the consideration of realistic spacecraft specifications and in a high-fidelity dynamical framework. The methodology and results here presented ultimately prepare the space community for potential launch opportunities to the Sun-Earth Lagrange points that could enable the low-cost exploration of small planetary bodies.

The baseline and requirements for the mission are introduced in Section 2, the selection of potential target asteroids is described in Section 3, the methodology for the design of high-impulse trajectories is described in Section 4, the design of low-thrust trajectories is described in Section 5, results are shown and discussed in Section 6, and further discussion can be found in Section 7.

\section{Mission baseline and requirements}

The first and second Sun-EMB Lagrange points have been extensively used by scientific missions to study the Sun and outer space. A total of seven missions were placed in an orbit around L1 since ISEE-3 in 1983 [19-25], and four missions around L2 since WMAP in 2001 [26-29]. Now that CubeSat capabilities enable more ambitious mission concepts, a future scientific mission to L1 or L2 may be leveraged to deploy a CubeSat capable of performing its own maneuvers to flyby a near-Earth asteroid. In case a dedicated launch to L1/L2 is not available, planned scientific missions for the 2020 decade include Aditya-1 to L1 [10], and JWST, WFIRST, Euclid, ARIEL and PLATO to L2 [11-15]. Halo and Lissajous orbits around L1 and L2 are most commonly used by this kind of missions, and missions such as Euclid, ARIEL and PLATO will moreover be launched into free-insertion transfer trajectories to L2 [13-15].

Any of these launch opportunities may enable a CubeSat to reach the Lagrange points and eventually depart towards an asteroid. The analysis contained in this paper is, however, only concerned with the cost of reaching a near-Earth asteroid after departing from L1 or L2. Analysis of the transfer phase to the Lagrange points is not included here, but implications and further requirements to perform these transfers are briefly discussed in Section 7.3. In particular, halo orbits are employed as the baseline departure condition for the asteroid flyby trajectories, provided that their equal inand out-of-plane periodicities allow for a more general analysis than Lissajous orbits. And orbits of equivalent size to those commonly used by scientific missions are considered [11,13,14,21,30]: 500,000 km x 1,500,000 km x 800,000 $\mathrm{km}$.

The CubeSat trajectory after departure from L1 or L2 until the encounter with the asteroid is additionally constrained to last less than 150 days. This is done in order to confine environmental implications of long-duration deep-space travel and the demand on spacecraft subsystems. For reference, a more detailed discussion on this topic can be found in the authors' previous work [18]. The specific time window considered here for departure from L1 and L2 spans between 
years 2019 and 2030, although the trajectory design methodology described in Sections 4 and 5 can be directly extended to other time windows.

In addition, due to the limited maneuverability and controllability of CubeSats, Earth and Moon gravity-assist maneuvers are discarded for the simplicity of the mission concept. JAXA's PROCYON microsatellite, for instance, attempted an Earth gravity assist in 2015 but an engine failure led to mission loss [8]. Lunar gravity-assist maneuvers using CubeSats, on the other hand, will be first demonstrated by missions such as EQUULEUS [31] and NEA Scout [32,33], but these maneuvers are also discarded in the analysis. Particularly, only departure trajectories away from the Earth are considered here, leveraging the natural instability of the initial halo orbits to depart from L1 and L2, and exploiting a simple mission concept that could potentially be operated autonomously.

A total $\Delta \mathrm{V}$ of $80 \mathrm{~m} / \mathrm{s}$ is assumed for the design of high-impulse and low-thrust trajectories. Numerous CubeSat propulsion systems with this capability are readily available or currently under development [34], and could be included within a 3 U CubeSat configuration along with the science payload and other spacecraft subsystems [18]. Larger CubeSat platforms could potentially have larger propulsive capabilities as well. A 3U CubeSat platform is considered here in contrast to previous studies of asteroid flyby missions using small spacecraft, such as the 6U NEA Scout mission or the 50-kg PROCYON mission [8,32,33].

As examples, the high-impulse thruster considered is VACCO's 0.5U, 5-mN warm-gas Propulsion Unit for CubeSats [35], and the low-thrust thruster is Busek's $0.4 \mathrm{U}, 100-\mu \mathrm{N}$ electrospray BET-100 propulsion system [36]. Both thrusters have reported $\Delta \mathrm{V}$ capabilities of $85 \mathrm{~m} / \mathrm{s}$ for a $3 \mathrm{U}$ CubeSat, and their specific impulses are 70 seconds and 2300 seconds, respectively. VACCO's high-impulse PUC, in particular, requires heavy duty cycling for proper thermal control: 20 minutes operating at maximum capability require 20 minutes off for cool-down (D. Carroll at CU Aerospace, personal communication, April 23, 2018). Such constraint is also considered in the design of high-impulse trajectories in Section 4.

It is also worth mentioning that, although the specific examples of VACCO's and Busek's thrusters are used for the mission, the methodology is applicable to any generic CubeSat (or SmallSat) mission from L1 or L2, as common features for this kind of missions are small $\Delta \mathrm{V}$ capabilities, small thrust magnitudes, duty cycling, etc. Additionally, Section 6 provides a discussion on how results vary depending on the total available $\Delta \mathrm{V}$ and the total mission duration.

The science payload on board the CubeSat is also likely to be tightly constricted in terms of mass and volume. Instrument performance at such small scales is limited, and therefore targeting a sufficiently large asteroid could facilitate a successful science campaign. Hence, a minimum threshold of approximately 100 meters in diameter is also imposed on the target asteroid for significant science return (absolute magnitude $H \leq 22.5$ ). A more in-depth discussion on the topic can also be found in [18]. Relatively large asteroids are also likely to have been observed from Earth for longer periods of time, and their trajectories are thus likely to be better characterized [37]. This would also facilitate the navigation campaign prior to the asteroid flyby.

Lastly, in the scenario that the CubeSat is launched along a larger mission, the position along the halo orbit at the departure epoch is assumed to be dictated by the launch opportunity and not controllable by the CubeSat (also discussed in Section 7.1). In order to provide some insight into the $\Delta V$ requirements as a function of orbit phasing, flyby trajectories are computed from nine departure points along the halo orbit equally spread over one period. Illustration of these predetermined departure points can be found in Section 6.

General requirements for the mission under consideration are summarized in Table 1.

\section{Table 1}

Mission requirements.

\begin{tabular}{|c|c|c|}
\hline & Requirement & Description \\
\hline 1. & $\begin{array}{l}\text { Launch opportunity to halo orbit around } \\
\text { Sun-EMB L1/L2 }\end{array}$ & $\begin{array}{l}\text { CubeSat is initially parked around the Sun-Earth barycentric Lagrange } \\
\text { points between years } 2019 \text { and } 2030\end{array}$ \\
\hline 2. & 150-day mission duration & $\begin{array}{l}\text { Asteroid flyby trajectory should last less than } 150 \text { days after departure } \\
\text { from L1/L2 halo orbit }\end{array}$ \\
\hline 3. & $80-\mathrm{m} / \mathrm{s}$ available $\Delta \mathrm{V}$ & $\begin{array}{l}\text { (a) High-impulse propulsion unit: VACCO’s } 0.5 \mathrm{U}, 5-\mathrm{mN} \text { PUC [35] } \\
\text { (b) Low-thrust propulsion unit: Busek's } 0.4 \mathrm{U}, 100-\mu \mathrm{N} \text { BET-100 [36] }\end{array}$ \\
\hline 4. & $\begin{array}{l}\text { Target asteroid larger than } 100 \text { meters in } \\
\text { diameter }\end{array}$ & $\begin{array}{l}\text { Desired asteroid size for proper observation during flyby and significant } \\
\text { scientific return }\end{array}$ \\
\hline
\end{tabular}




\section{Target selection}

Potential target asteroids are identified by first approximating the region that can be reached by a CubeSat from L1 and L2, and then selecting near-Earth asteroids whose trajectories pass close to that reachable region. This pruning process is performed in the CR3BP dynamical model in consideration of the gravitational influences of the Sun and the Earth-Moon barycenter only.

Due to the limited $\Delta \mathrm{V}$ available on a CubeSat, departure trajectories from halo orbits are strongly driven by the natural dynamics around the Lagrange points. Departure trajectories consequently remain close to those on the unstable invariant manifolds. Taking into account the maximum mission length (150 days) and available $\Delta \mathrm{V}(80 \mathrm{~m} / \mathrm{s})$, the accessible region from any given point along the halo orbit is approximated by: (1) implementing an initial $80-\mathrm{m} / \mathrm{s}$ impulsive maneuver along the unstable direction associated to that point, and (2) propagating the resulting trajectory over 150 days. These 150-day trajectories are herein referred to as "approximated unstable invariant manifolds."

Asteroids larger than 100 meters flying within 0.1 astronomical units from the Earth between years 2019 and 2030 are identified through JPL's Center for Near-Earth Object Studies website [38]. Their ephemerides are then obtained using JPL's HORIZONS telnet interface [39], and only asteroids whose trajectories pass within 0.02 au from the accessible region are finally selected as potential targets. As further discussed in Section 6, larger distances from the reachable region do not allow for an asteroid encounter given the limited $\Delta \mathrm{V}$. Thus, this 0.02 -au threshold reduces the number of asteroids to which flyby trajectories are attempted to be computed.

For completeness, the equations of motion in the CR3BP and in the ephemeris model are summarized in Eq. (1) and Eq. (2), respectively.

$$
\begin{gathered}
\frac{d v_{x}^{S}}{d \tau}=\frac{d^{2} x^{S}}{d \tau^{2}}=-(1-\mu) \cdot\left(x^{S}+\mu\right) / d^{3}-\mu \cdot\left(x^{S}-1+\mu\right) / r^{3}+x^{S}+2 v_{y}^{S} \\
\frac{d v_{y}^{S}}{d \tau}=\frac{d^{2} y^{S}}{d \tau^{2}}=-(1-\mu) \cdot y^{S} / d^{3}-\mu \cdot y^{S} / r^{3}+y^{S}-2 v_{x}^{S} \\
\frac{d v_{z}^{S}}{d \tau}=\frac{d^{2} z^{S}}{d \tau^{2}}=-(1-\mu) \cdot z^{S} / d^{3}-\mu \cdot z^{S} / r^{3} \\
\frac{d \boldsymbol{v}^{I}}{d t}=\frac{d^{2} \boldsymbol{r}^{I}}{d t^{2}}=-G \sum_{i=1}^{n} m_{i} \cdot\left(\boldsymbol{r}^{I}-\boldsymbol{r}_{i}^{I}\right) /\left|\boldsymbol{r}^{I}-\boldsymbol{r}_{i}^{I}\right|^{3}
\end{gathered}
$$

where the gravitational influences considered in the ephemeris-model analysis are those of the Sun, EMB, Mars, Jupiter and Saturn.

\section{High-impulse asteroid flyby trajectories}

Preliminary design of high-impulse trajectories is generally performed under the assumption that changes in velocity can be generated instantaneously. However, as mentioned in Section 4, current propulsion systems for CubeSats can only generate small thrust magnitudes and may require heavy duty cycling. For the specific case of VACCO's 5-mN thruster, approximately 35 hours are required to generate its total $80 \mathrm{~m} / \mathrm{s}$ of $\Delta \mathrm{V}$. Due to these long thrusting times, the impulsive maneuver model may be used for a preliminary analysis, but its validity shall be verified. The design of impulsive maneuvers is introduced in Section 4.1. If higher levels of fidelity are desired, a more realistic maneuver model shall be considered and is introduced in Section 4.2: quasi-impulsive model. This higher-fidelity model assumes that maneuvers are composed of 20-minute continuous thrust arcs ( $5 \mathrm{mN}$ in magnitude and fixed in direction) followed by 20 minutes off duty for engine cool-down.

\subsection{Impulsive trajectories}


The goal in the current section is to design one- or two-impulse trajectories that will allow a CubeSat to depart from its initial halo orbit and flyby a near-Earth asteroid.

The problem is initially solved here in the time-independent CR3BP model. With the goal of minimizing the required $\Delta \mathrm{V}$, a total of nine unknowns are present in this optimization problem: the time of the second maneuver, $t_{\Delta v_{2}}$, components of each impulsive maneuver, $\Delta \boldsymbol{v}_{1}$ and $\Delta \boldsymbol{v}_{2}$, the time of the asteroid encounter, $t_{\text {enc }}$, and the total time of flight, TOF. In an effort to determine whether an asteroid is ever reachable from the predetermined departure points, the time of encounter and the TOF are selected as two independent optimizations variables, which effectively defines the departure epoch as a free variable. Once asteroid accessibility is determined in the CR3BP, the departure epoch is set as a fixed initial condition in the ephemeris-model analysis, for comparison of $\Delta \mathrm{V}$ and TOF requirements for a given departure epoch. Therefore, only the time of encounter or the TOF remain as optimization variables in the ephemeris model. The optimization problem can therefore be posed as in Eq. (3).

$$
\begin{aligned}
\text { find: } & \Delta \boldsymbol{v}_{1}, \Delta \boldsymbol{v}_{2}, t_{\Delta \boldsymbol{v}_{2}}, t_{\text {enc }} \text { and/or TOF } \\
\text { to minimize: } & \left|\Delta \boldsymbol{v}_{1}\right|+\left|\Delta \boldsymbol{v}_{2}\right| \\
\text { subject to: } & \text { Eq. (1) or Eq. (2) with impulses } \Delta \boldsymbol{v}_{1} \text { at } t=0 \text { and } \Delta \boldsymbol{v}_{2} \text { at } t=t_{\Delta \boldsymbol{v}_{2}} \\
& \left|\Delta \boldsymbol{v}_{1}\right|+\left|\Delta \boldsymbol{v}_{2}\right| \leq 80 \mathrm{~m} / \mathrm{s} \\
& \text { TOF } \leq 150 \text { days }
\end{aligned}
$$

A similar optimization problem was faced during Chang'e-2's extended mission [17], where the optimization variables were: the time of departure, time of the second maneuver, time of the asteroid encounter, and components of each impulsive maneuver (a total of nine variables). This nonlinear parametric optimization problem was solved using sequential quadratic programming in a time-dependent restricted four-body dynamical model (with gravitational influences of the Sun, Earth and Moon).

The optimization problem is solved in this paper using a genetic algorithm in combination with a state-transition matrix differential corrector [40]. The genetic algorithm is used to determine three optimization variables: the time of the second maneuver, and the magnitude of both impulsive maneuvers. The sum of both maneuvers is limited to $80 \mathrm{~m} / \mathrm{s}$ according to mission requirements. In particular, the open-source tool NSGA-II [41] is used to solve this part of the problem. The STM differential corrector is then applied on each individual of the GA to determine the remaining variables: direction of both impulsive maneuvers (i.e., two angles to describe each maneuver), the time of encounter, and the TOF. The optimization problem in the ephemeris model is posed and solved in the same way as in the CR3BP model (described in Eq. (3)), except for the time of encounter and the TOF that are explicitly related once the departure epoch is set as an initial condition.

The STM is used in the differential corrector to linearly approximate the variation in final position due to the variation in direction of the initial velocity, and in the TOF (according to Eq. (4), in combination with Eq. (5)). Through the differential corrector, the direction of each maneuver and TOF are thus iterated to achieve a final position as close as possible to the target point. The target point is also iterated within the differential corrector and selected as the point on the trajectory of the asteroid closest to trajectory of the CubeSat in the previous iteration. This target selection process finally determines the time of encounter according to the epoch of the asteroid trajectory.

The initial guess of this differential corrector process is generated with an initial maneuver along the unstable direction associated to the departure point (whose magnitude is determined by the GA), and with a TOF of 150 days. Finally, if the asteroid can be reached with a single maneuver at a lower cost than with two maneuvers, then the second maneuver is not implemented.

The relationship between variations in the final state due to variations in the initial state and in the TOF can be linearly approximated in terms of the STM as in Eq. (4).

$$
\delta x\left(t_{f}\right)=\left[\underline{\phi}\left(t_{f}, t_{0}\right), \frac{d x\left(t_{f}\right)}{d t}\right] \cdot\left[\begin{array}{c}
\delta x\left(t_{0}\right) \\
\delta \mathrm{TOF}
\end{array}\right]
$$

Consider now that the components of the velocity vector can be expressed in terms of two angles and its magnitude: for instance, the elevation with respect to the $x-y$ plane, and the azimuth with respect to the $x$-axis on the $x$-y plane, such 
as: $\boldsymbol{v}(t)=|\boldsymbol{v}(t)| \cdot[\cos (\varepsilon(t)) \cos (\theta(t)), \cos (\varepsilon(t)) \sin (\theta(t)), \sin (\varepsilon(t))]^{T}$. Variations in the final state can thus be related to variations in the initial direction of the velocity applying the chain rule in Eq. (5).

$$
\begin{aligned}
& \underline{\boldsymbol{\phi}}_{x\left(t_{f}\right) / \varepsilon\left(t_{0}\right)}=\underline{\boldsymbol{\phi}}_{x\left(t_{f}\right) / \boldsymbol{v}\left(t_{0}\right)} \cdot \frac{d \boldsymbol{v}\left(t_{0}\right)}{d \varepsilon\left(t_{0}\right)} \\
& \underline{\boldsymbol{\phi}}_{x\left(t_{f}\right) / \theta\left(t_{0}\right)}=\underline{\boldsymbol{\Phi}}_{x\left(t_{f}\right) / \boldsymbol{v}\left(t_{0}\right)} \cdot \frac{d \boldsymbol{v}\left(t_{0}\right)}{d \theta\left(t_{0}\right)}
\end{aligned}
$$

Additionally, the STM is numerically propagated from time $t_{0}$ to time $t_{f}$ according to the differential equation in Eq. (6), with the identity matrix as its initial condition, and with the Jacobian matrix $\underline{\boldsymbol{A}}(t)$ defined as the partial derivatives of the EoM of the corresponding dynamical system with respect to the state vector.

$$
\frac{d \underline{\phi}\left(t, t_{0}\right)}{d t}=\underline{\boldsymbol{A}}(t) \cdot \underline{\boldsymbol{\phi}}\left(t, t_{0}\right)
$$

Trajectories in the ephemeris model are computed following the same procedure as in the CR3BP, but with the additional constraint in the departure epoch. The departure epoch from the halo orbit is specifically set as: $t_{d e p}=t_{\text {enc }}-$ $\mathrm{TOF}_{\mathrm{CR} 3 \mathrm{BP}}$, where $t_{\text {enc }}$ and $\mathrm{TOF}_{\mathrm{CR} 3 \mathrm{BP}}$ are those of the corresponding CR3BP trajectories. This ephemeris-model analysis is performed to compare $\triangle \mathrm{V}$ and TOF requirements to those in the CR3BP for a given departure time, and to verify that the flyby opportunities identified in the CR3BP model also exist in a higher-fidelity dynamical framework. As such, ephemeris-model trajectories are computed only for those asteroids that are reachable in the CR3BP model, and only from the departure points from which these asteroids are accessible.

Additionally, it is worth noticing that perfectly periodic halo orbits do not appear in a real ephemeris dynamical model and only quasi-periodic orbits can be computed. For reference, a methodology to compute quasi-periodic orbits in the ephemeris model can be found in previous work by Gómez et al. [42]. These quasi-periodic orbits are epoch dependent, and thus the CubeSat's position and velocity at the time of departure from L1/L2 would depend on some particular conditions at the time of orbit insertion. In the interest of generality, the departure conditions in the ephemeris-model analysis are not computed assuming a mission-dependent quasi-periodic orbit, but simply expressing the departure position and velocity along the CR3BP halo orbit in an inertial reference frame (at the corresponding departure epoch). This simplified approach results in departure conditions that dynamically are close but differ from the states along a quasiperiodic orbit. As a consequence, $\Delta \mathrm{V}$ requirements are expected to slightly differ from those along a quasi-periodic orbit, but are considered sufficient for the purpose of verifying CR3BP flyby trajectories also appear in the ephemeris model and for comparing $\mathrm{TOF}$ and $\Delta \mathrm{V}$ requirements.

\subsection{Quasi-impulsive trajectories}

The higher-fidelity quasi-impulsive maneuver model is introduced to consider the long thrusting times and duty cycling required by VACCO's thruster to generate its total $\triangle \mathrm{V}$. Here maneuvers are modeled as multiple 20-minute continuous thrust arcs ( $5 \mathrm{mN}$ in magnitude and fixed in direction) with 20-minute gaps in between thrust arcs. It is however worth mentioning that, although the values used in this analysis are specific to VACCO's thruster, the methodology is applicable to all high-impulse CubeSat thrusters of small thrust magnitude and requiring duty cycling.

Each 20-min thrust arc is assumed to be fixed in direction with respect to an inertial reference frame to reduce the demand on the CubeSat's attitude control system. Thrust arcs in the CR3BP analysis are also assumed to be fixed in direction, provided the negligible rotation of the CR3BP synodic reference frame with respect to an inertial reference frame within 20 minutes (approximately 0.014 degrees). Trajectories are designed with the impulsive trajectories described in Section 4.1 as reference, first in the CR3BP model and then in a high-fidelity ephemeris model.

The transition from impulsive trajectories to quasi-impulsive trajectories is performed through yet another intermediate maneuver model. This intermediate model considers maneuvers are composed of multiple smaller impulses executed every 40 minutes instead, according to duty-cycling constraints. Effectively, this intermediate model is used as an approximation of the higher-fidelity quasi-impulsive model composed of 20-minute thrust arcs, leveraging the approach proposed by Sims and Flanagan [43] to approximate low-thrust trajectories through multiple-impulse trajectories.

The time of execution of the intermediate-model impulses is determined by the time of execution of the two maneuvers along the reference impulsive trajectory: for the first impulsive maneuver, smaller impulses are executed every 40 minutes past the reference maneuver; and for the second impulsive maneuver, half of the smaller impulses are executed 
before the reference maneuver, and half of them are executed after (see Fig. 2 for an illustration of how impulses are distributed). The magnitude of these smaller impulses is limited to $1.5 \mathrm{~m} / \mathrm{s}$, which is the $\Delta \mathrm{V}$ magnitude that can be generated within 20 minutes by VACCO's $5-\mathrm{mN}$ thruster on a 4-kg spacecraft. And the number of smaller impulses is then calculated in proportion to the $\Delta \mathrm{V}$ magnitude of each impulsive maneuver on the reference trajectory.

Two approaches are implemented to obtain the direction of these multiple smaller impulses. A first approach employs an STM differential corrector on each impulse to target points along the reference impulsive trajectory until the last impulse allows to reach the asteroid (employing Eq. (4) and Eq. (5) as in Section 4.1). A second approach is further developed in Appendix 2 and leverages the solution to the linearized optimization problem to reach a certain target (i.e., the asteroid) through multiple impulses along the trajectory. The goal of these two approaches is effectively to shadow the reference impulsive trajectory, and therefore the same asteroid encounter point as in the impulsive trajectory is selected (which also determines the time of encounter). In the ephemeris-model analysis, the departure time is additionally constrained and set equal to $t_{d e p}=t_{e n c}-\mathrm{TOF}_{\mathrm{CR} 3 \mathrm{BP}}$ (as in Section 4.1). Multiple-impulse trajectories in the ephemerismodel thus have predetermined $t_{d e p}$ and $t_{e n c}$, and hence, they are constrained to have the same TOF as the impulsive trajectories. Nonetheless, results in Section 6.2 show that similar $\Delta \mathrm{V}$ requirements to those of impulsive trajectories can be found despite constraining the time of encounter and the TOF.

In addition to these two approaches, a continuation method is also employed in cases where a smoother transition between the impulsive and the intermediate models is required. Starting from the reference impulsive trajectory until a trajectory with multiple $1.5-\mathrm{m} / \mathrm{s}$ impulses executed every 40 minutes is obtained, the continuation method exploits: (a) trajectories with impulses of $1.5 \mathrm{~m} / \mathrm{s}$ executed every 5 minutes, 10 minutes, 20 minutes, etc., (b) trajectories with impulses executed every 40 minutes with magnitude $15 \mathrm{~m} / \mathrm{s}, 10 \mathrm{~m} / \mathrm{s}, 5 \mathrm{~m} / \mathrm{s}$, etc., or a combination of approaches (a) and (b). Due to gravity losses and the suboptimality of performing multiple smaller impulses instead of two large impulsive maneuvers, it is also necessary in some cases to include additional $1.5-\mathrm{m} / \mathrm{s}$ impulses until the encounter with the asteroid is achieved (generally not more than two).

Once trajectories with this intermediate model are obtained, quasi-impulsive trajectories are computed employing the multiple $1.5-\mathrm{m} / \mathrm{s}$ impulse trajectories as reference. Each 20-min thrust arc is initiated at the execution time of the $1.5-\mathrm{m} / \mathrm{s}$ impulses (see Fig. 2). The magnitude of the thrust is limited to $5 \mathrm{mN}$ according to the specifications of the thruster, and the fixed direction of each thrust arc is also determined through an STM differential corrector.

In this case, the EoM are modified to include the acceleration and change in mass due to the thrust (see Eq. (7)), and the state vector is augmented for the STM to include information on the variations in the final state due to variations in the thrust components (Eq. (8)).

$$
\begin{aligned}
\left(\frac{d \boldsymbol{v}}{d t}\right)_{\text {thrust }} & =\left(\frac{d \boldsymbol{v}}{d t}\right)_{\text {cruise }}+\boldsymbol{T} / m \\
\frac{d m}{d t} & =-|\boldsymbol{T}| /\left(I_{s p} \cdot g_{0}\right)
\end{aligned}
$$

where the unpowered EoM are those described in Eq. (1) and Eq. (2).

The thrust components (constant throughout time) are included in an augmented state vector, and the STM is also augmented to relate variations in the final state to variations in the thrust components (see Eq. (8)).

$$
\begin{aligned}
\boldsymbol{x}_{A} & =\left[x, y, z, v_{x}, v_{y}, v_{z}, m, T_{x}, T_{y}, T_{z}\right]^{T} \\
\underline{\boldsymbol{\phi}}_{A}\left(t_{f}, t_{0}\right) & =\left[\underline{\boldsymbol{\phi}}_{\boldsymbol{x}_{A}}\left(t_{f}\right) / \boldsymbol{r}\left(t_{0}\right), \underline{\boldsymbol{\phi}}_{\boldsymbol{x}_{A}}\left(t_{f}\right) / \boldsymbol{v}\left(t_{0}\right), \underline{\boldsymbol{\phi}}_{\boldsymbol{x}_{A}}\left(t_{f}\right) / m\left(t_{0}\right), \underline{\boldsymbol{\phi}}_{\boldsymbol{x}_{A}}\left(t_{f}\right) / \boldsymbol{T}\left(t_{0}\right)\right]
\end{aligned}
$$

The augmented STM is propagated in time as in Eq. (6), although now the Jacobian matrix shall also include partial derivatives of the state vector with respect to the thrust components. Finally, a chain-rule transformation such as that in Eq. (5) is applied to relate variations in the final state to variations in the direction of the thrust vector. Through these augmented EoM and STM, the differential corrector is employed to determine the direction of the thrust required to reach a certain target.

For the design of quasi-impulsive trajectories, the STM differential corrector targets points along the reference trajectory (i.e., the intermediate-model trajectory) until the last thrust arc allows to reach the asteroid. The same encounter point as in the impulsive and multiple-impulse trajectories is targeted, and therefore the three types of trajectories have 
common time and point of encounter. In the ephemeris-model analysis, and as a consequence of fixing the time of departure, the three types of trajectories have a common TOF as well. Finally, it is also worth mentioning that a continuation method is not necessary to transition between the multiple-impulse and the quasi-impulsive trajectories.

Illustrated in Fig. 2 are the three maneuver models employed in Sections 4.1 and 4.2, including the intermediate model that facilitates the transition between the impulsive and quasi-impulsive trajectories.

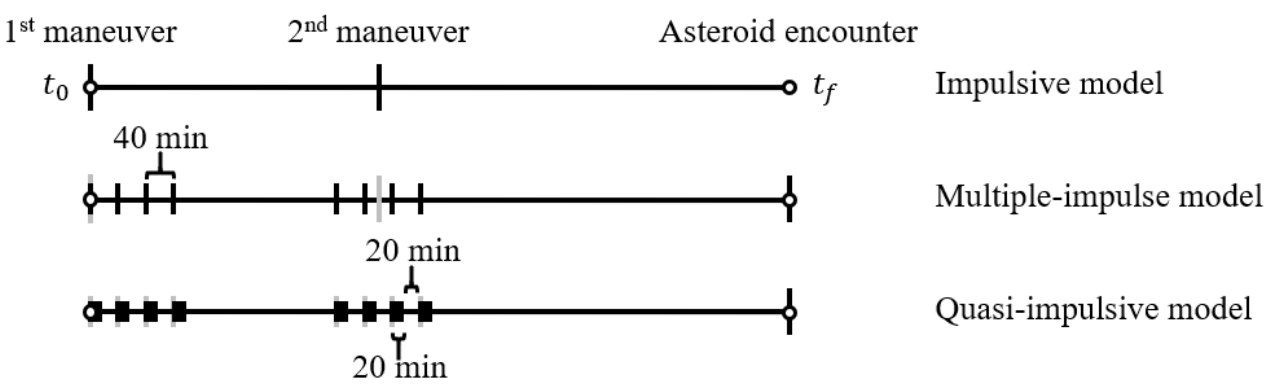

Fig. 2. Impulsive, intermediate and quasi-impulsive maneuver models.

\section{Low-thrust asteroid flyby trajectories}

In contrast to the high-impulse trajectories computed in Section 4, low-thrust trajectories result from exerting a continuous thrust force over an extended period of time, non-negligible in comparison to the overall length of the mission.

In this work, low-thrust flyby trajectories are calculated following a two-step process: (1) a suboptimal initial guess is generated through a genetic algorithm in combination with an STM differential corrector, and (2) the initial guess is refined by a direct optimization solver. A modified version of GPOPS [44] implemented with IPOPT [45] is used to perform step (2). The same procedure is followed to design trajectories first in the CR3BP dynamical model and then in the ephemeris model.

The suboptimal initial guess is generated in a similar manner to the impulsive trajectories in Section 4.1, with the goal of maximizing the final mass. The final mass is constrained to be larger than the CubeSat's dry mass: $m_{d r y}=4 \mathrm{~kg}-$ $m_{\text {prop }}$. The total propellant mass is deduced from the specific impulse and total $\Delta \mathrm{V}$ of Busek's BET-100 propulsion system [36], and is equivalent to $80 \mathrm{~m} / \mathrm{s}$ of $\Delta \mathrm{V}$. These trajectories are now composed of one or two low-thrust arcs along the trajectory, of fixed direction and constant magnitude. The magnitude of these thrust arcs is set equal to $100 \mu \mathrm{N}$, according to the thruster's specifications.

A total of nine optimization variables are present in the CR3BP optimization problem: the duration of each thrust arc, $\Delta t_{\boldsymbol{T}_{1}}$ and $\Delta t_{\boldsymbol{T}_{2}}$, the direction of each thrust arc, $\left(\varepsilon_{\boldsymbol{T}_{1}}, \theta_{\boldsymbol{T}_{1}}\right)$ and $\left(\varepsilon_{\boldsymbol{T}_{2}}, \theta_{\boldsymbol{T}_{2}}\right)$, the time of execution of the second thrust arc, $t_{\boldsymbol{T}_{2}}$, the time of encounter, $t_{\text {enc }}$, and the time of flight, TOF. The fixed direction of each thrust arc is described by its corresponding elevation and azimuth angles. Additionally, in the ephemeris-model analysis, the departure epoch from the halo orbit is set as in Section 4.1: $t_{d e p}=t_{\text {enc }}-\mathrm{TOF}_{\mathrm{CR} 3 \mathrm{BP}}$. Fixing the departure time therefore explicitly relates the TOF and the time of encounter in the ephemeris-model analysis, and only one of the variables remains as an unknown. The low-thrust optimization problem is described in Eq. (9).

$$
\begin{aligned}
\text { find: } & \left(\varepsilon_{\boldsymbol{T}_{1}}, \theta_{\boldsymbol{T}_{1}}\right),\left(\varepsilon_{\boldsymbol{T}_{2}}, \theta_{\boldsymbol{T}_{2}}\right), \Delta t_{\boldsymbol{T}_{1}}, \Delta t_{\boldsymbol{T}_{2}}, t_{\boldsymbol{T}_{2}}, t_{\text {enc }} \text { and/or TOF } \\
\text { to minimize: } & -m\left(t_{f}\right) \\
\text { subject to: } & \text { Eq. (1) or Eq. (2) with } d m / d t=0 \text { during cruise } \\
& \text { Eq. (7) during thrust arcs } \boldsymbol{T}_{1} \text { at } t \in\left[0, \Delta t_{\boldsymbol{T}_{1}}\right] \text { and } \boldsymbol{T}_{2} \text { at } t \in\left[t_{\boldsymbol{T}_{2}}, t_{\boldsymbol{T}_{2}}+\Delta t_{\boldsymbol{T}_{2}}\right] \\
& m\left(t_{f}\right) \geq m_{d r y} \\
& \text { TOF } \leq 150 \text { days }
\end{aligned}
$$

The genetic algorithm is in this case employed to determine three variables: the duration of each thrust arc, and the time of execution of the second thrust arc. The STM differential corrector is implemented as in Section 4.2 to include information on the variations in the final state due to variations in the thrust direction. As such, it employs the augmented 
EoM and STM described in Eq. (7) and Eq. (8), and the chain rule described in Eq. (5). The remaining optimization variables are obtained through this STM differential corrector: the direction of each thrust arc, the time of encounter, and the TOF. Selection of the position of encounter (which determines $t_{\text {enc }}$ ) is performed as in Section 4.1: it is iterated within the differential corrector as the closest point on the trajectory of the asteroid to the trajectory of the CubeSat in the previous iteration.

Once a suboptimal initial guess is generated, the optimal low-thrust trajectories are obtained through a direct optimization solver (GPOPS). The direct optimization solver is used in combination with the interior-point optimizer IPOPT to: (1) discretize the trajectory into segments, (2) approximate the state and control variables (thrust components) within each segment via Lagrange interpolating polynomials, (3) transcribe the equations of the optimal control problem into a nonlinear programming problem, and (4) solve the NLP problem.

The optimization problem solved by GPOPS is the same as that defined in Eq. (9), but using as optimization variables the thrust components of each thrust arc instead. Six time-varying optimization variables are thus considered now: $\boldsymbol{T}_{1}(t)$ and $\boldsymbol{T}_{2}(t)$ subject to $\left|\boldsymbol{T}_{1}(t)\right| \leq 100 \mu \mathrm{N}$ and $\left|\boldsymbol{T}_{2}(t)\right| \leq 100 \mu \mathrm{N}$, rather than the fixed direction of the thrust arcs: $\left(\varepsilon_{\boldsymbol{T}_{1}}, \theta_{\boldsymbol{T}_{1}}\right)$ and $\left(\varepsilon_{\boldsymbol{T}_{2}}, \theta_{\boldsymbol{T}_{2}}\right)$. The solution provided by GPOPS is therefore a discretized solution to the optimal control problem, in which the direction and magnitude of the thrust arcs are no longer constrained to be constant as in the suboptimal initial guess. Assessment of this optimal solution can finally be performed through forward propagation of the EoM and a linear or quadratic approximation of the thrust components within each thrust arc.

It is also worth mentioning that the same asteroid encounter point as in the suboptimal initial guess is targeted by the direct optimization solver (i.e., same $t_{\text {enc }}$ ). This last constraint enforces optimal low-thrust trajectories designed in the ephemeris model to have the same TOF as the suboptimal initial guess, as both the time of encounter and time of departure are predetermined. This additional constraint is imposed here as it simplifies the declaration of the optimization problem within the direct optimization solver. Although low-thrust trajectories with slightly lower $\Delta \mathrm{V}$ costs may exist to other encounter points, this approach still serves the purpose of comparing optimal low-thrust $\Delta \mathrm{V}$ requirements to those of suboptimal low-thrust and high-impulse trajectories. Lastly, it is also recommended to pose the optimization problem in non-dimensional units to improve the convergence properties of the solver.

\section{Results and discussion}

\subsection{Target selection}

The target selection process described in Section 3 begins by identifying as potential targets only asteroids with an Earth encounter within 0.1 au from the Earth. Out of those potential targets, a second step in the pruning process is selecting asteroids whose trajectories pass within 0.02 au from the approximated unstable invariant manifolds. This second step allows to reduce the number of asteroids to which flyby trajectories are attempted to be computed. As an example and illustrated in Fig. 3 (represented in the Sun-EMB synodic reference frame, centered on the Earth, and projected on the $x-y$ plane), it is observed how impulsive asteroid encounter trajectories remain in fact close to those on the approximated unstable invariant manifold. The encounters with asteroid $2010 \mathrm{JG}$ in Fig. 3 occur within 0.05 au from the Earth, and at a distance of 0.003 au from the approximated manifold trajectories. These distances are well within the selection criteria of $0.1 \mathrm{au}$ and $0.02 \mathrm{au}$. All encounter opportunities identified in the following sections occur as well at a maximum distance of $0.065 \mathrm{au}$ from the Earth, and at most at 0.016 au from the approximated manifold trajectories.

Conclusively and as suggested in Section 3, the region that can be reached by a CubeSat-due to their modest propulsive capabilities - can be approximated by the unstable invariant manifold associated to the departure halo orbits.

For reference, 774 near-Earth asteroids pass within 0.1 au from the Earth between years 2019 and 2030, of which approximately 200 pass within 0.02 au from the approximated unstable invariant manifolds, and computation of flyby trajectories is finally possible to 41 of those asteroids. Impulsive flyby opportunities to these 41 asteroids in the CR3BP and ephemeris models are summarized in Appendix 1. 


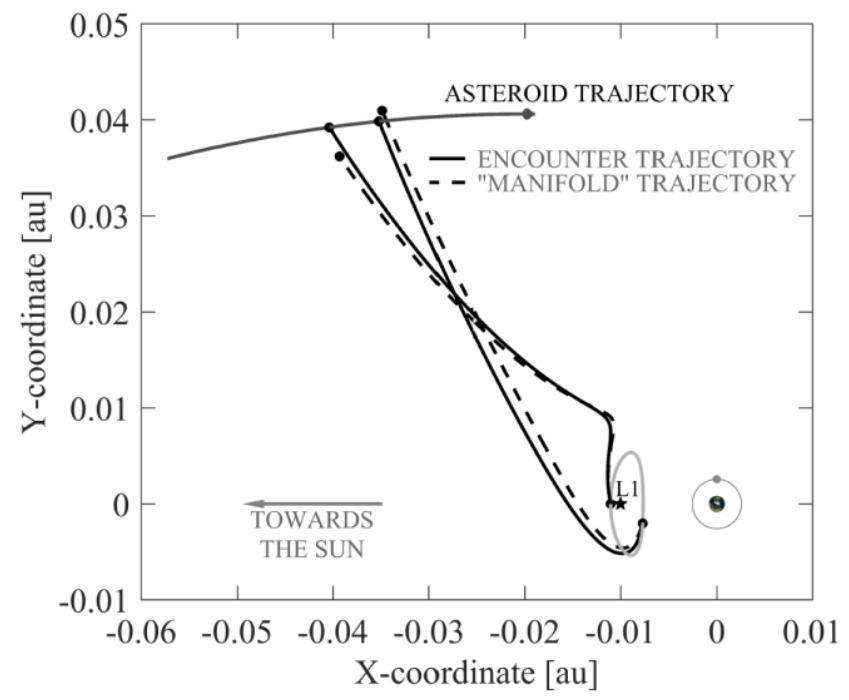

Fig. 3. CR3BP asteroid encounter and (approximated) unstable-invariant-manifold trajectories (asteroid $2010 \mathrm{JG}$ ).

\subsection{High-impulse asteroid flyby trajectories}

The number of reachable asteroids from L1 and L2 as a function of the calendar year are displayed in Table 2. Flyby opportunities are observed to be common and appear almost every year, except for years 2020 and 2025 from L1. A future flyby mission using CubeSats could potentially piggyback along ESA missions PLATO and ARIEL [13,14], for instance, which are scheduled for launch to L2 in the late 2020s. During these years, at least one asteroid is reachable every year from L2, with up to 5 reachable asteroids in 2028.

Table 2

Number of reachable asteroids per year from Sun-EMB L1 and L2 (CR3BP model, impulsive trajectories).

\begin{tabular}{|c|c|c|c|c|c|c|c|c|c|c|c|}
\hline \multirow{2}{*}{$\begin{array}{l}\text { Reachable asteroids } \\
\text { from }\end{array}$} & \multicolumn{11}{|c|}{ Year } \\
\hline & 2019 & 2020 & 2021 & 2022 & 2023 & 2024 & 2025 & 2026 & 2027 & 2028 & 2029 \\
\hline L1 & 1 & 0 & 3 & 4 & 3 & 1 & 0 & 1 & 2 & 3 & 2 \\
\hline L2 & 3 & 3 & 1 & 2 & 1 & 1 & 1 & 1 & 2 & 5 & 1 \\
\hline
\end{tabular}

The mission concept described in Section $\overline{2}$ assumes certain mission characteristics such as the maximum mission length and the propulsion system. In support of a more general mission study, Fig. 4 and Fig. 5 illustrate the number of reachable asteroids from $\mathrm{L} 1$ and $\mathrm{L} 2$ as a function of the required TOF and available $\Delta \mathrm{V}$. Flyby trajectories in this analysis are designed with the goal of minimizing the $\Delta \mathrm{V}$, and it is observed in Fig. 4 that nearly half of the asteroids are reached through trajectories of $\mathrm{TOF} \geq 125$ days. Longer mission durations thus improve asteroid accessibility and this shall be considered in the mission analysis. Similarly, it is observed in Fig. 5 that the number of reachable asteroids consistently increases with the available $\Delta \mathrm{V}$, and this illustration may provide an orientative estimation of the number of reachable asteroids (within 150 days) for different $\Delta \mathrm{V}$ capabilities. 


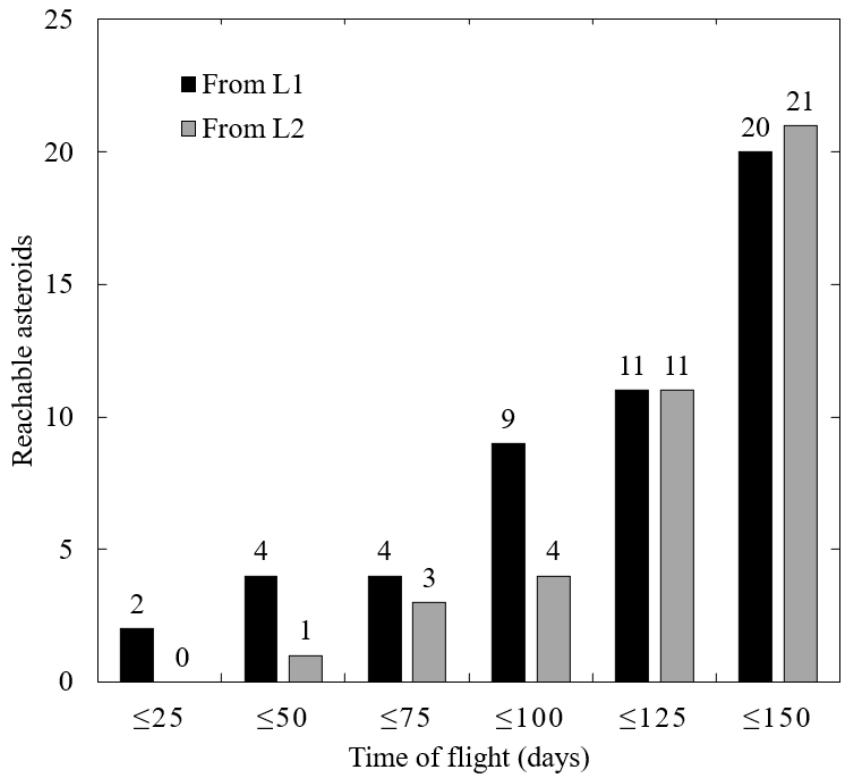

Fig. 4. Cumulative number of reachable asteroids from L1 and L2 as a function of TOF (CR3BP model, impulsive trajectories, $\Delta \mathrm{V} \leq 80 \mathrm{~m} / \mathrm{s})$.

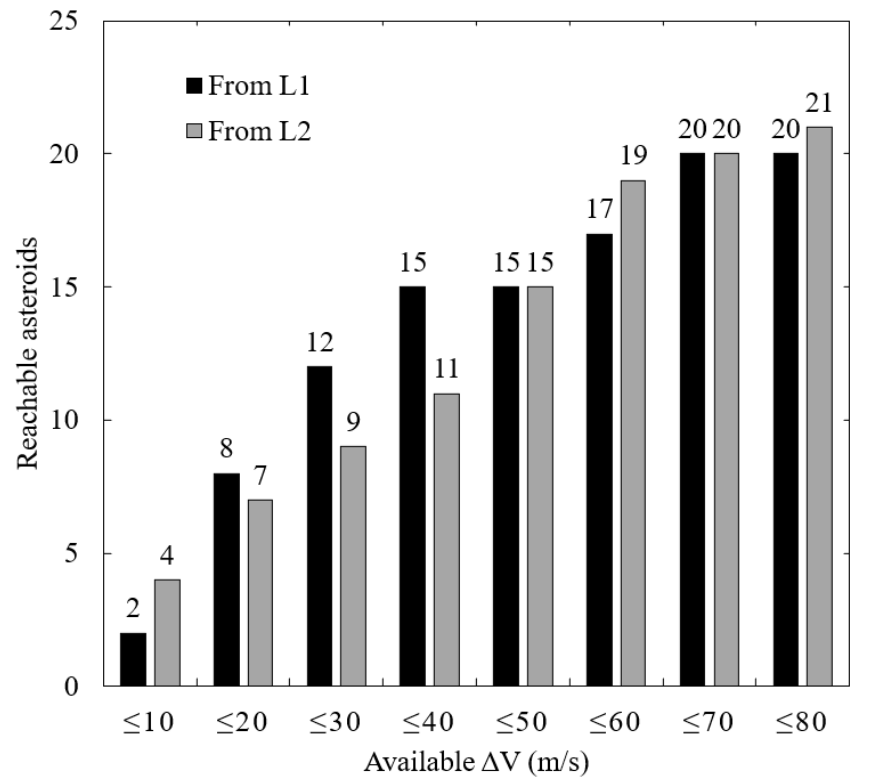

Fig. 5. Cumulative number of reachable asteroids from $\mathrm{L} 1$ and L2 as a function of available $\triangle \mathrm{V}$ (CR3BP model, impulsive trajectories, $\mathrm{TOF} \leq 150$ days).

Flyby trajectories are computed from several departure points along the halo orbits as mentioned in Section 2. As an example, Fig. 6 illustrates the CR3BP impulsive $\Delta \mathrm{V}$ and TOF requirements to encounter asteroid $2011 \mathrm{GA}$ from L1 (represented in the Sun-EMB synodic reference frame and projected on the $x-y$ plane). This particular asteroid has a close encounter with Earth on October 15, 2023, and can be reached from only 7 out of the 9 predetermined departure points considered. The $\Delta \mathrm{V}$ requirement ranges from $24.5 \mathrm{~m} / \mathrm{s}$ to $53.2 \mathrm{~m} / \mathrm{s}$, and the TOF ranges from 99.3 days to 150 days, depending on the departure point.

The design of impulsive trajectories in the CR3BP is meaningful as it provides an effective tool to determine asteroid accessibility (i.e., whether an asteroid can be reached from a certain departure point) and to estimate TOF requirements in more sophisticated dynamical and thrusting models (e.g., ephemeris model, quasi-impulsive model, low-thrust model). Initial estimation of $\Delta \mathrm{V}$ requirements can also be obtained through the CR3BP model, however, results may differ significantly depending on the model. Illustrated in Fig. 7 are the requirements to reach the same asteroid in the ephemeris model, using an impulsive maneuver model. It is observed how asteroid $2011 \mathrm{GA}$ can be reached from the same departure points as in the CR3BP analysis, and the $\triangle \mathrm{V}$ and TOF requirements are comparable. Although there is a clear correlation between the $\Delta \mathrm{V}$ requirements in the CR3BP and ephemeris models, substantial differences between the two models appear in some cases.

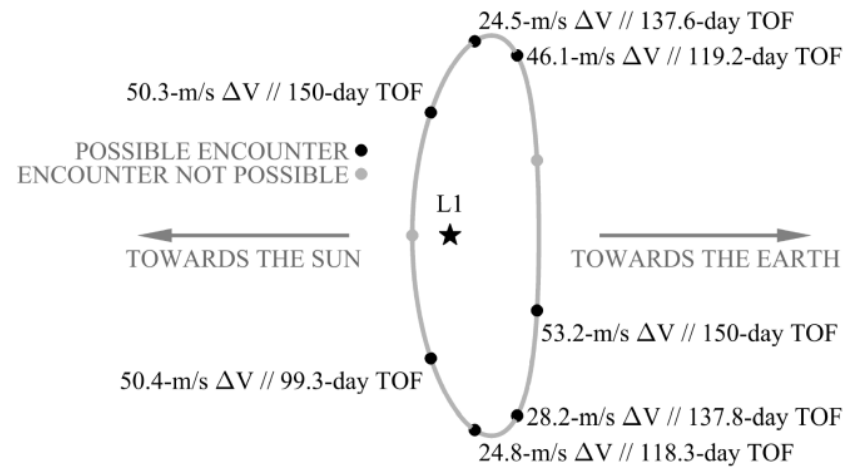

Fig. 6. $\Delta \mathrm{V}$ and TOF requirements to reach asteroid $2011 \mathrm{GA}$ along L1 halo orbit (CR3BP model, impulsive trajectories).

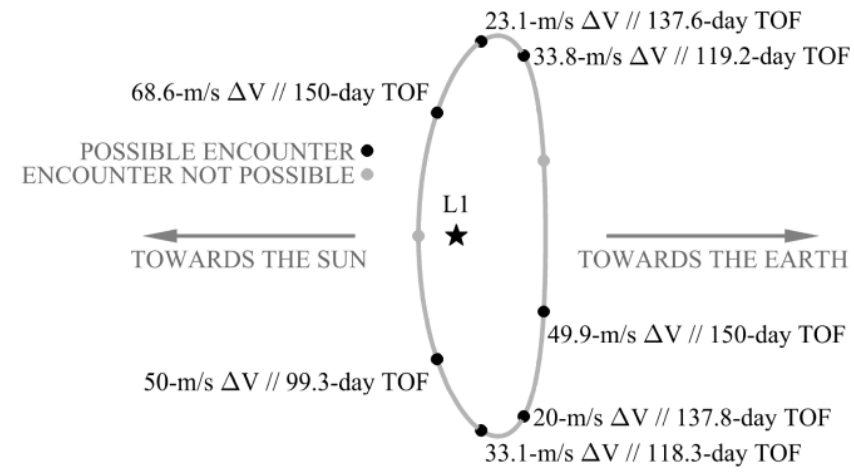

Fig. 7. $\Delta \mathrm{V}$ and $\mathrm{TOF}$ requirements to reach asteroid $2011 \mathrm{GA}$ along L1 halo orbit (ephemeris model, impulsive trajectories). 
A list of $\Delta \mathrm{V}$ and TOF requirements to reach the same 41 asteroids in the ephemeris model are also provided in Appendix 1. The following conclusions can be drawn from the results in the ephemeris model, as compared to those in the CR3BP model:

- If the asteroid is reachable in the CR3BP, an impulsive flyby trajectory can also be computed in the ephemeris model (in approximately $95 \%$ of the cases): for some of the asteroids, it is not possible to compute a flyby trajectory from some of the departure points with the available $\Delta \mathrm{V}$ and TOF.

- The $\Delta \mathrm{V}$ requirement in the ephemeris model differs by up to $\pm 15 \mathrm{~m} / \mathrm{s}$ from the CR3BP requirement in approximately $80 \%$ of the cases. The difference may be up to $\pm 30 \mathrm{~m} / \mathrm{s}$.

- The TOF in the CR3BP and ephemeris models agree within \pm 0.2 days.

Conclusively, the CR3BP analysis does provide accurate estimation in terms of TOF and asteroid accessibility, however, differences of $\pm 15 \mathrm{~m} / \mathrm{s}$ in $\Delta \mathrm{V}$ do represent a large percent difference considering the available $\Delta \mathrm{V}(80 \mathrm{~m} / \mathrm{s})$. Geometric representations of flyby trajectories in the two models show minimal differences and thus illustrations of these trajectories are not included here.

Section 4.2 described the methodology to transition from the lower-fidelity impulsive maneuver model to a higherfidelity quasi-impulsive maneuver model. The latter incorporates duty cycling and non-instantaneous maneuvers into the model. Computation of quasi-impulsive trajectories shows that, even if CubeSat propulsion systems have low thrust magnitudes and require heavy duty cycling, an impulsive maneuver model still holds as a valid estimation of $\Delta \mathrm{V}$ and TOF requirements. As an example and for comparison with Fig. 6 and Fig. 7, illustrated in Fig. 8 and Fig. 9 are the $\Delta$ V and TOF required to reach asteroid $2011 \mathrm{GA}$ using the quasi-impulsive maneuver model.

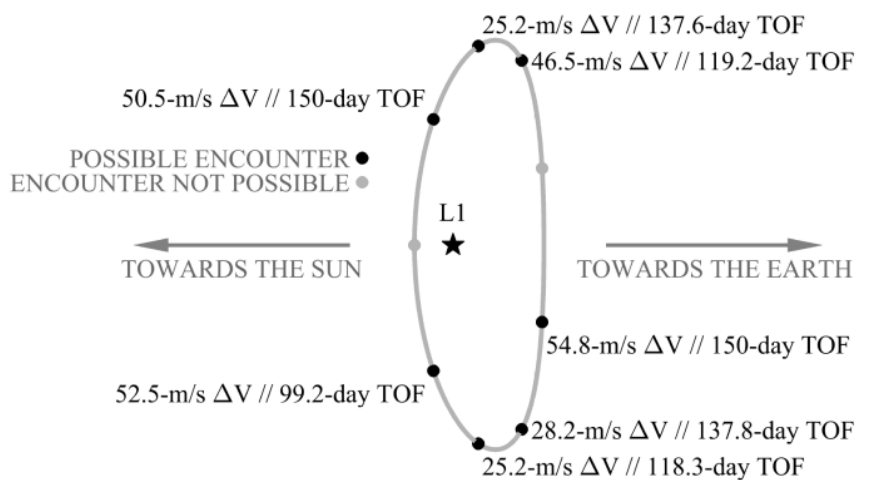

Fig. 8. $\Delta$ V and TOF requirements to reach asteroid $2011 \mathrm{GA}$ along L1 halo orbit (CR3BP model, quasi-impulsive trajectories).

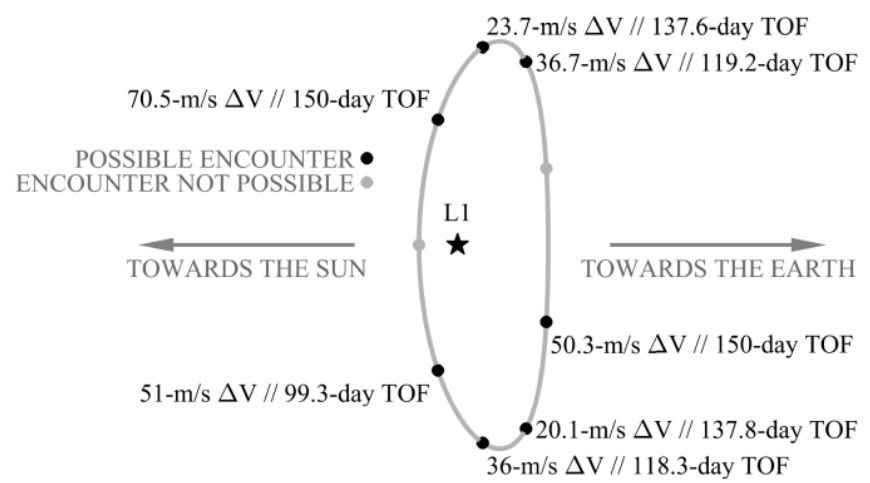

Fig. 9. $\Delta \mathrm{V}$ and TOF requirements to reach asteroid $2011 \mathrm{GA}$ along L1 halo orbit (ephemeris model, quasi-impulsive trajectories).

Similarly, Table 3 summarizes the level of agreement between the impulsive and intermediate maneuver models, and between the intermediate and quasi-impulsive maneuver models. Conclusively, analysis of the results for the 41 reachable asteroids shows that quasi-impulsive $\Delta \mathrm{V}$ requirements can be estimated through the impulsive maneuver model with an accuracy better than $3 \mathrm{~m} / \mathrm{s}$ in the CR3BP model and better than $4 \mathrm{~m} / \mathrm{s}$ in the ephemeris model (most cases better than $1.5 \mathrm{~m} / \mathrm{s}$ and $2.5 \mathrm{~m} / \mathrm{s}$, respectively). It is worth recalling that quasi-impulsive trajectories in the ephemeris model are constrained to have the same TOF as impulsive trajectories (discussed in Section 4.2). This additional constraint justifies the slightly higher $\Delta \mathrm{V}$ increment in the ephemeris model than that in the CR3BP. Lastly, geometric representations of impulsive and quasi-impulsive trajectories show nearly no distinction, and thus these trajectories are not illustrated in this paper.

Table 3

$\Delta \mathrm{V}$ and TOF comparison for high-impulse trajectories.

\begin{tabular}{|c|c|c|c|}
\hline \multicolumn{2}{|c|}{$\begin{array}{l}\text { Intermediate maneuver model relative to } \\
\text { impulsive trajectories }\end{array}$} & \multicolumn{2}{|c|}{$\begin{array}{l}\text { Quasi-impulsive trajectories relative to } \\
\text { intermediate maneuver model }\end{array}$} \\
\hline CR3BP model & Ephemeris model & CR3BP model & Ephemeris model \\
\hline $\begin{array}{l}\text { - Flyby trajectories can be } \\
\text { computed in all cases. }\end{array}$ & $\begin{array}{l}\text { - Flyby trajectories can be } \\
\text { computed in all cases. }\end{array}$ & $\begin{array}{l}\text { - Flyby trajectories can be } \\
\text { computed in all cases. } \\
-\Delta \mathrm{V} \text { may vary } \pm 0.1 \mathrm{~m} / \mathrm{s} \text {. }\end{array}$ & $\begin{array}{l}\text { - Flyby trajectories can be } \\
\text { computed in all cases. } \\
-\Delta V \text { may vary } \pm 1 \mathrm{~m} / \mathrm{s} \text {. }\end{array}$ \\
\hline
\end{tabular}




\begin{tabular}{|c|c|c|c|}
\hline $\begin{array}{l}-\Delta \mathrm{V} \text { increment below } 1.5 \\
\mathrm{~m} / \mathrm{s} \text { in } 85 \% \text { of cases (at } \\
\text { most } 3 \mathrm{~m} / \mathrm{s} \text { ). } \\
\text { - TOF may vary } \pm 0.1 \text { days. }\end{array}$ & $\begin{array}{l}-\Delta \mathrm{V} \text { increment below } 1.5 \\
\mathrm{~m} / \mathrm{s} \text { in } 80 \% \text { of cases (at } \\
\mathrm{most} 3 \mathrm{~m} / \mathrm{s}) . \\
\text { - Same TOF (fixed). }\end{array}$ & - TOF may vary \pm 0.1 days. & - Same TOF (fixed). \\
\hline
\end{tabular}

\subsection{Low-thrust asteroid flyby trajectories}

Low-thrust trajectories are designed according to the methodology described in Section 5. A first step in the process is generating an initial guess composed of one or two low-thrust arcs of fixed direction and constant magnitude. This suboptimal initial guess is then provided to a direct optimization solver to refine the solution.

A first observation in this optimization process is that computation of an initial guess fulfilling the TOF and $\Delta \mathrm{V}$ requirements may not always be possible, even if a feasible impulsive trajectory was computed from the same departure point. Two possible explanations are provided for these cases: either (1) a feasible low-thrust solution does not exist, or (2) a feasible solution exists but the suboptimal initial guess is not good enough given the fixed-direction constraint imposed on the thrust arcs.

If an initial guess can be generated, it is also observed that the optimal solution is always composed of thrust arcs of maximum magnitude: bang-bang control law. Furthermore, the optimal direction of the thrust arcs changes almost linearly with time and can be approximated through linear polynomials for assessment of the solution.

For comparison with the impulsive requirements in Fig. 6 and Fig. 7, optimal low-thrust $\Delta \mathrm{V}$ and TOF requirements to reach asteroid 2011 GA from L1 are illustrated in Fig. 10 and Fig. 11. And again, geometric representations of impulsive and low-thrust trajectories show minimal differences and thus they are not illustrated here.

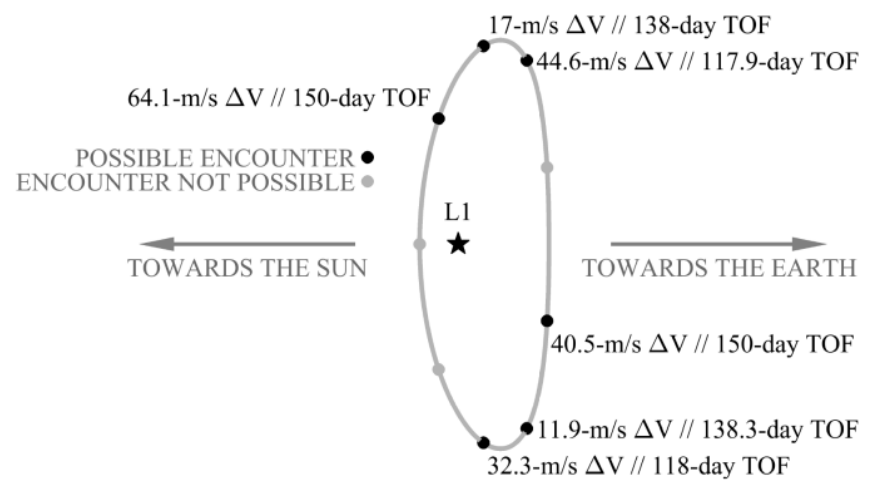

Fig. 10. $\Delta \mathrm{V}$ and TOF requirements to reach asteroid $2011 \mathrm{GA}$ along L1 halo orbit (CR3BP model, optimal low-thrust trajectories).

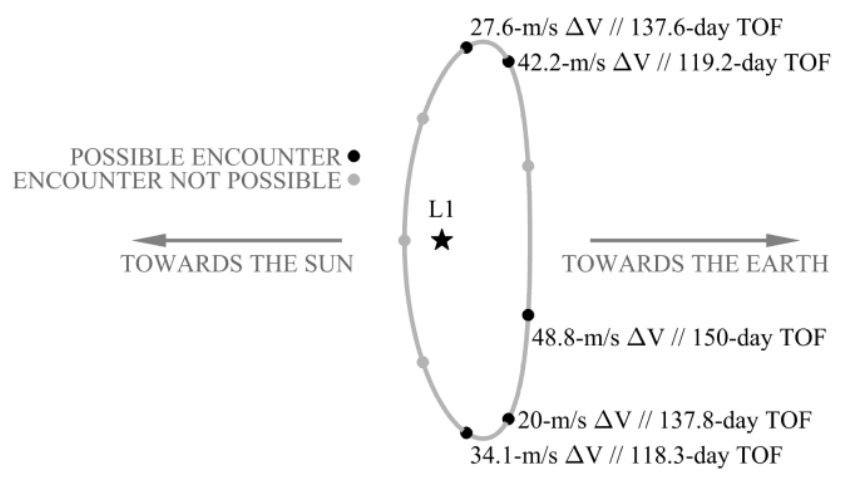

Fig. 11. $\Delta V$ and TOF requirements to reach asteroid $2011 \mathrm{GA}$ along L1 halo orbit (ephemeris model, optimal low-thrust trajectories).

Optimal low-thrust trajectories also have comparable $\Delta \mathrm{V}$ costs to impulsive trajectories, but $\Delta \mathrm{V}$ requirements may differ significantly between the two thrusting models. In the CR3BP model, the optimal low-thrust cost may be anywhere between $-20 \mathrm{~m} / \mathrm{s}$ and $+15 \mathrm{~m} / \mathrm{s}$ from the $\Delta \mathrm{V}$ value of impulsive trajectories. And in the ephemeris model, $\Delta \mathrm{V}$ results differ within $\pm 15 \mathrm{~m} / \mathrm{s}$ with respect to the impulsive result. Furthermore, the low-thrust TOF in the CR3BP analysis may vary \pm 10 days with respect to the impulsive TOF. In the ephemeris model, on the other hand, the TOF of low-thrust and impulsive trajectories agree within \pm 0.3 days, as a result of predetermining the departure epoch in the ephemeris-model analysis. It is also worth highlighting that low-thrust trajectories with a lower $\Delta \mathrm{V}$ requirement than impulsive trajectories are obtained in some cases. Impulsive trajectories, however, are expected to offer the lowest $\Delta \mathrm{V}$ solution. This discrepancy is due to the inability of the genetic algorithm to find the global minimum when computing some of the impulsive trajectories.

As a summary of the low-thrust optimization process, Table 4 provides a $\Delta \mathrm{V}$ and TOF comparison between the impulsive and suboptimal low-thrust trajectories, and between the suboptimal and optimal low-thrust solutions.

Table 4

$\Delta V$ and TOF comparison for low-thrust trajectories.

\begin{tabular}{lcll}
\hline $\begin{array}{l}\text { Suboptimal initial guess relative to } \\
\text { impulsive trajectories }\end{array}$ & \multicolumn{2}{l}{$\begin{array}{l}\text { Optimal low-thrust solution relative to } \\
\text { suboptimal initial guess }\end{array}$} \\
\hline CR3BP model & Ephemeris model & CR3BP model & Ephemeris model \\
\hline
\end{tabular}


- Flyby trajectories cannot be computed in $15 \%$ of cases.

- $\Delta \mathrm{V}$ varies $\pm 20 \mathrm{~m} / \mathrm{s}$ in $85 \%$

of cases (at most $+30 \mathrm{~m} / \mathrm{s}$ ).

- TOF may vary \pm 10 days.
- Flyby trajectories cannot be computed in $20 \%$ of cases.

$-\Delta \mathrm{V}$ varies $\pm 15 \mathrm{~m} / \mathrm{s}$ in $85 \%$ of cases (at most $+45 \mathrm{~m} / \mathrm{s}$ ).

- TOF may vary \pm 0.3 days.
- Flyby trajectories cannot be computed in all cases.

- $\Delta \mathrm{V}$ reduction $<10 \mathrm{~m} / \mathrm{s}$ in

$90 \%$ of cases (up to $40 \mathrm{~m} / \mathrm{s}$ ).

$(\Delta \mathrm{V}$ varies $[-20$ to +15$] \mathrm{m} / \mathrm{s}$

relative to impulsive result).

- TOF may vary \pm 1.5 days.
- Flyby trajectories can be computed in all cases.

$-\Delta \mathrm{V}$ reduction $<10 \mathrm{~m} / \mathrm{s}$ in

$90 \%$ of cases (up to $30 \mathrm{~m} / \mathrm{s}$ ).

$(\Delta \mathrm{V}$ varies $\pm 15 \mathrm{~m} / \mathrm{s}$

relative to impulsive result).

- Same TOF (fixed).

Conclusively, the analysis of impulsive trajectories provides an orientative estimation of low-thrust requirements, but a specific analysis for low-thrust trajectories shall be performed if more accurate results are desired, both in the CR3BP and in the ephemeris model. The suboptimal initial guess also provides a reasonable estimation of the optimal $\Delta \mathrm{V}$ requirement: the suboptimal low-thrust cost is generally less than $10 \mathrm{~m} / \mathrm{s} \mathrm{higher} \mathrm{than} \mathrm{the} \mathrm{optimal} \mathrm{low-thrust} \Delta \mathrm{V}$ requirement. Lastly, and as a potential improvement to this initial guess, allowing the thrust arcs to have a varying direction (e.g., linear or quadratic) may allow to find an initial guess in cases where a feasible solution exists but a solution with thrust arcs of fixed direction is not found.

\section{Further discussion}

\subsection{Phasing cost}

Results in Section 6 show that each asteroid can only be reached from certain regions along the halo orbit. The $\mathrm{Cu}$ beSat must then be within one of those regions at the required departure epoch to reach the target asteroid. It is therefore worth investigating the $\Delta \mathrm{V}$ required to modify the phase of the CubeSat in case its original phase after reaching the Lagrange points is not suitable to encounter a certain asteroid.

A potential solution to modifying the phase of the CubeSat is transferring to another halo orbit of longer or shorter period (henceforth referred to as "secondary halo orbit"). After a certain amount of time, the CubeSat could then return to the original halo orbit with a phase different to its original and suitable for the flyby, or directly depart from the secondary halo orbit.

Previous work by Gómez et al. [46] discussed the cost of transferring between halo orbits around the first Sun-EMB Lagrange point. Using a linear approximation of the problem, transfer costs are initially estimated as a function of the normalized $z$-amplitude of the halo orbit. Halo orbits used by previous and future scientific missions $[11,13,14,16,21]$ are characterized by a $\beta$ parameter of the order of 0.2 (non-dimensional units), with associated cost of $\sim 700 \mathrm{~m} / \mathrm{s}$ to produce a unit change in $\beta: \Delta \beta=1$. Current propulsion systems, however, can only generate approximately $80 \mathrm{~m} / \mathrm{s}$ of $\Delta \mathrm{V}$, which translates into transfers of at most $\Delta \beta \sim 0.1$.

Given that halo orbits around the Sun-EMB Lagrange points are characterized by a similar periodicity of approximately 180 days, a change in amplitude of $\Delta \beta \sim 0.1$ yields a change in period of only $\sim 0.5$ days. Therefore, the phase change that can be achieved through a secondary halo orbit, and employing all of the available $\Delta \mathrm{V}$, is approximately of only 2 degrees per calendar year. Conclusively, it is a reasonable assumption to consider the CubeSat will not be able to significantly modify its own phase, and thus its departure position from the halo orbit and corresponding epoch will be dictated by the available launch opportunity.

\subsection{Likelihood of flyby opportunities}

Given the probable infeasibility of controlling the CubeSat's phase along the halo orbit, it is worth investigating the likelihood of a flyby opportunity to appear if the CubeSat reaches the Lagrange point at an arbitrary phase along the halo orbit. Only a preliminary assessment of this problem is performed here, and it is based on the results presented in Section 6.

In this paper, flyby trajectories are computed from nine predetermined departure points along the halo orbits, and it is observed that asteroids can be reached only from some of those departure points. For the purpose of this preliminary analysis, it is further assumed that a particular asteroid can be reached if the CubeSat is anywhere within the neighborhood of those access points at the required departure epoch. Illustrated in Fig. 12 are the regions along the halo orbit from which an asteroid is assumed to be reachable, based on whether the asteroid can be reached from the predetermined departure points. 


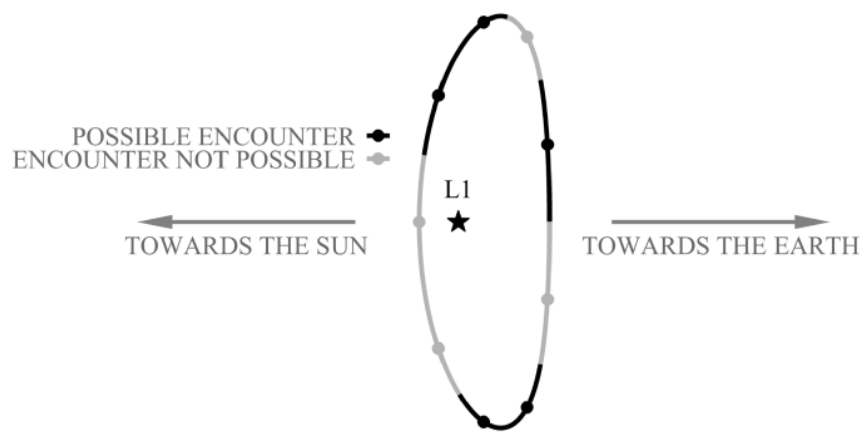

Fig. 12. Sample diagram of assumed asteroid accessibility from halo orbit.

Assuming that, at a certain epoch, the CubeSat is deployed at an arbitrary position along the halo orbit, the likelihood for at least one asteroid to be reachable within a certain calendar year is summarized in Table 5. It is observed that this likelihood greatly varies depending on the calendar year: from $0 \%$ in years where no asteroid opportunities were identified, to $70 \%$ in years where multiple asteroids can be reached from multiple departure points.

Table 5

Yearly likelihood of a flyby opportunity to arise from Sun-EMB L1 and L2 (CR3BP model, impulsive trajectories).

\begin{tabular}{|c|c|c|c|c|c|c|c|c|c|c|c|c|}
\hline \multirow{2}{*}{$\begin{array}{l}\text { Reachable } \\
\text { asteroids from }\end{array}$} & \multicolumn{11}{|c|}{ Year } & \multirow[b]{2}{*}{ Mean } \\
\hline & 2019 & 2020 & 2021 & 2022 & 2023 & 2024 & 2025 & 2026 & 2027 & 2028 & 2029 & \\
\hline L1 & $22.2 \%$ & $0.0 \%$ & $52.5 \%$ & $70.5 \%$ & $49.6 \%$ & $22.2 \%$ & $0.0 \%$ & $25.7 \%$ & $40.4 \%$ & $62.1 \%$ & $39.6 \%$ & $35.0 \%$ \\
\hline L2 & $53.1 \%$ & $48.3 \%$ & $22.2 \%$ & $39.2 \%$ & $29.1 \%$ & $27.7 \%$ & $11.1 \%$ & $26.4 \%$ & $45.3 \%$ & $64.4 \%$ & $17.9 \%$ & $35.0 \%$ \\
\hline
\end{tabular}

Table 5 shows an average likelihood of $35 \%$ for at least one flyby opportunity to arise within a certain year, for an arbitrary predetermined phase. If the CubeSat could, instead, remain in the halo orbit for two calendar years before departure, the average likelihood of a flyby opportunity to appear within those two years is of 55\%. If the asteroid could remain in the halo orbit for three year before departure, the average likelihood is then $71 \%$, and so on.

It is therefore clear that, unless the CubeSat could remain in the halo orbit for several years, the likelihood of flyby opportunities to appear is greatly dependent on the phase condition when inserted into the halo orbit.

\subsection{Further mission requirements and considerations}

Analysis in this paper shows that short-transfer asteroid flyby trajectories are possible even with the very limited $\Delta \mathrm{V}$ capabilities of CubeSats. However, depending on the available launch opportunity, the total $\Delta \mathrm{V}$ requirement for the mission may not only be limited to the cost of the asteroid flyby, but may need to include: (a) the $\Delta \mathrm{V}$ to reach the orbit around L1/L2, (b) station-keeping costs around the L1/L2 orbit, and (c) the $\Delta \mathrm{V}$ for correction maneuvers along the asteroid flyby trajectory.

As for (a), potential piggyback opportunities along ESA's ARIEL or PLATO [13,14], for instance, could place the CubeSat into a free-insertion transfer trajectory to $\mathrm{L} 1 / \mathrm{L} 2$. The $\Delta \mathrm{V}$ required to reach its orbit around $\mathrm{L} 1 / \mathrm{L} 2$ would thus be limited to corrections of the perigee velocity and of launcher dispersions. As an example, Herschel mission proved that this kind of transfers are possible with only $10 \mathrm{~m} / \mathrm{s}$ of $\Delta \mathrm{V}$ [16]. The cost of these transfers, however, may be higher depending on launcher performance and on how early maneuvers can be performed (dependent on orbit determination). Consequently, future missions Euclid, ARIEL and PLATO have allocated up to $50 \mathrm{~m} / \mathrm{s}$ of $\Delta \mathrm{V}$ to accomplish their transfers to L2 [13-15].

The cost of (b), on the other hand, is generally limited to $1-3 \mathrm{~m} / \mathrm{s}$ per year as reported by previous missions to the Lagrange points [47], and similar station-keeping budgets are allocated for future scientific missions [13-15]. If the CubeSat is expected to remain around its L1/L2 orbit until a flyby opportunity arises, then this additional $\Delta \mathrm{V}$ demand shall be included in the mission budget as well.

Lastly, the required $\Delta \mathrm{V}$ for the correction maneuvers along the asteroid flyby trajectory may be estimated with analyses similar to that in [18]. The preliminary analysis in [18] shows that flyby accuracies are largely driven by the available $\Delta \mathrm{V}$, and at least $20 \mathrm{~m} / \mathrm{s}$ may be necessary for an autonomous CubeSat to achieve flyby altitudes of the order of hundreds of kilometers. 
In conclusion, depending on the target asteroid and on the launch opportunity, the total $\Delta \mathrm{V}$ requirement for the mission could nearly double the $\sim 80 \mathrm{~m} / \mathrm{s}$ that can be provided by current CubeSat propulsion systems on a $3 \mathrm{U}$ platform. Challenges for the mission thus comprise an excessive $\Delta \mathrm{V}$ demand, a total mission duration of potentially several years, operations and navigation challenges to reach and maintain the L1/L2 orbit, and others.

In regards to the mission duration, short transfer times to the asteroids are considered here, but a waiting time of several years around L1/L2 may still be required until a flyby opportunity appears. A system capable of withstanding long-term deep-space environmental conditions would then be required, which may further increase the complexity of the spacecraft design. As examples, other deep-space CubeSat missions such as 6U NEA Scout [33] or 12U M-ARGO [48] also have expected lifetimes of several years.

As for navigation, a particularly critical phase of the mission would be the transfer trajectory to L1/L2. Time-sensitive correction maneuvers must be performed after deployment from the launch vehicle, and thus accurate orbit determination must be available in a timely manner (e.g., 2-5 days after deployment). As such, radiometric observations may be required to successfully complete this phase. Station-keeping around L1/L2 may require correction maneuvers every 30 days, similarly to large scientific missions $[14,15]$. In the interest of a low-cost mission, it would be advantageous to autonomously perform orbit determination around L1/L2, to minimize ground support. As an example, the 12U LUMIO mission is expected to autonomously navigate around the Earth-Moon L2 point [49]. Further analysis, however, shall be performed to determine whether autonomy can also be achieved around the Sun-EMB Lagrange points and with a smaller spacecraft. Lastly, analysis such as that in [18] showed that autonomous navigation along the asteroid flyby trajectory can be performed without support from ground antennas.

Although many are the challenges to the mission concept specified in Section 2, similar mission scenarios could still be proven viable through further analysis or relaxing some of the mission constraints. A few alternatives are summarized here:

- Further navigation and guidance analysis of the asteroid flyby trajectories may result in lower $\Delta \mathrm{V}$ costs for the correction maneuvers. Analysis in [18] exclusively considered a fully-autonomous optical navigation scenario and a simplistic guidance strategy consisting of only one correction maneuver before the flyby. Possible improvements could be achieved through, for instance, several correction maneuvers along the trajectory or if some radiometric observations are available for navigation (thus abandoning the fully-autonomous scenario) [50].

- Longer than 150-day asteroid flyby trajectories can reduce the cost to encounter asteroids (refer to Fig. 4), increase the number of reachable asteroids, and increase the likelihood of flyby opportunities to appear. The waiting time around L1/L2 could in this way be reduced, but it will also increase the demand on some of the subsystems due to farther distances from Earth, and also on the navigation and guidance strategies due to longer uncertainty propagation times.

- More complex trajectory designs including Earth or Moon gravity-assist maneuvers may enable additional flyby opportunities although these maneuvers are yet to be demonstrated by CubeSats. Heteroclinic connections between halo orbits could also allow the CubeSat to transfer between the L1 and L2 points at virtually no cost [51], effectively combining the L1 and L2 yearly flyby likelihoods listed in Table 5 .

- A larger CubeSat or SmallSat platform could not only provide sufficient $\Delta \mathrm{V}$ to complete the different phases of the mission, but could also allocate more instruments (with possibly higher performance) to augment the potential scientific return of the mission.

As a final comment, the number of reachable asteroids and therefore the likelihood of flyby opportunities are as well expected to drastically increase with the discovery of near-Earth objects through new wide-field survey telescopes such as the Large Synoptic Survey Telescope. LSST, for instance, is to be implemented in 2020 and is projected to increase the number of known NEO population by a factor of 10 over its ten-year lifetime [52]. Numerous new asteroid flyby opportunities can therefore be expected to become available as the catalogue of NEOs is completed in the upcoming years.

\section{Concluding remarks}

CubeSat capabilities have rapidly evolved over the past years and could potentially provide a low-cost solution for planetary exploration. In view of the current interest in interplanetary CubeSats, asteroid flyby trajectories are designed in consideration of CubeSat-specific limitations: limited $\Delta \mathrm{V}$, small thrust magnitude, duty cycling, etc. The mission concept assumes a CubeSat is initially parked in a halo orbit around the Sun-Earth barycentric Lagrange points, from where flyby opportunities between years 2019 and 2030 are identified. 
A methodology to compute high-impulse and low-thrust flyby trajectories in the CR3BP and ephemeris models is presented, and the validity of the impulsive maneuver model is assessed. Results show that the CR3BP model provides an accurate estimation of the time of flight in a high-fidelity ephemeris model (within \pm 0.2 days); however, $\Delta \mathrm{V}$ requirements in the CR3BP model generally differ up to $\pm 15 \mathrm{~m} / \mathrm{s}$ with respect to those in the ephemeris model, which implies a substantial percent error compared to the available $\Delta \mathrm{V}$ considered $(80 \mathrm{~m} / \mathrm{s})$. It is also observed that, due to the modest propulsive capabilities of CubeSats, the region that can be reached from the departure halo orbits can be effectively approximated by trajectories on the unstable invariant manifolds.

A quasi-impulsive maneuver model is also implemented incorporating non-instantaneous changes in velocity and duty cycling. It is observed that an impulsive maneuver model still holds as a valid approach despite duty cycling and the long thrusting times required to produce the total $\Delta \mathrm{V}$. Results show that an impulsive maneuver model can estimate non-impulsive $\Delta \mathrm{V}$ requirements with an accuracy better than $4 \mathrm{~m} / \mathrm{s}$. Additionally, an impulsive maneuver model may also provide an initial estimation of low-thrust requirements, although low-thrust costs may be up to $15 \mathrm{~m} / \mathrm{s} \mathrm{higher}$.

Conclusively, the CR3BP model is shown to serve as an effective tool for a preliminary analysis: e.g., identification of flyby opportunities and orientative estimation of requirements in a high-fidelity ephemeris model. An impulsive maneuver model is also shown to be a valid approach for the computation of high-impulse trajectories (including noninstantaneous changes in velocity and duty cycling). If higher levels of fidelity are desired, however, analysis in a real ephemeris model and a specific analysis for low-thrust trajectories are required.

This paper therefore provides an extensive analysis of asteroid flyby opportunities using CubeSats in consideration of realistic thrusting and dynamical models. Flyby opportunities from the Sun-Earth barycentric Lagrange points are shown to be common, and multiple asteroids could be reached in the upcoming years with very low $\Delta \mathrm{V}$ requirements. These flyby opportunities are however contingent to the position and epoch in which the CubeSat reaches its halo orbit, and current propulsive capabilities may not suffice when considering other phases of the mission. As a conclusion, the complexity of the mission concept may need to increase, some mission constraints shall be relaxed, and further analysis may be required to improve the viability of the mission. Lastly, the number of potential target asteroids is also expected to dramatically increase as new wide-field survey telescopes such as LSST complete the catalogue of known near-Earth objects.

Although numerous challenges remain to be addressed, this work shows that CubeSats and SmallSats have the potential to leverage future launch opportunities to the Sun-Earth barycentric Lagrange points and significantly lower the cost of near-Earth asteroid exploration.

\section{References}

[1] J. Schoolcraft, A. Klesh, T. Werne, MarCO: Interplanetary Mission Development on a CubeSat Scale, in: AIAA SpaceOps Conference, 2016: pp. 1-8. doi:10.2514/6.2016-2491.

[2] A. Klesh, J. Krajewski, MarCO: CubeSats to Mars in 2016, in: 29th Annual AIAA/USU Small Satellite Conference, 2015: pp. 1-7.

[3] J. Singer, J. Pelfrey, G. Norris, Secondary Payload Opportunities on NASA's Space Launch System (SLS) Enable Science and Deep Space Exploration, in: Space Operations: Contributions from the Global Community, Springer, 2017: pp. 207-220. doi:10.1007/978-3-319-51941-8.

[4] European Space Agency, CubeSats Joining Hera Mission to Asteroid System, (2019). http://www.esa.int/Our_Activities/Operations/Space_Safety_Security/Hera/CubeSats_joining_Hera_mission_to _asteroid_system/(print) (accessed March 27, 2019).

[5] S. Watanabe, Y. Tsuda, M. Yoshikawa, S. Tanaka, T. Saiki, S. Nakazawa, Hayabusa2 Mission Overview, Space Science Reviews. 208 (2017) 1-14. doi:10.1007/s11214-017-0377-1.

[6] C. Greco, M. Di Carlo, L. Walker, M. Vasile, Analysis of NEOs Reachability with Nano-satellites and Low-thrust Propulsion, in: Small Satellites, Systems and Services Symposium, Sorrento, Italy, 2018. doi:10.5897/AJEST2016.2108.

[7] N. Strange, E. Asphaug, K. Grogan, D. Landau, K. Reh, Planetary Science Decadal Survey - Near Earth Asteroid Trajectory Opportunities in 2020-2024, Pasadena, CA, USA, 2010. 
[8] S. Campagnola, N. Ozaki, Y. Sugimoto, C.H. Tam, H. Chen, Y. Kawabata, S. Ogura, B. Sarli, Y. Kawakatsu, R. Funase, S. Nakasuka, Low-thrust Trajectory Design and Operations of PROCYON, the First Deep-Space MicroSpacecraft, in: International Astronautical Congress, Jerusalem, Israel, 2015.

[9] J.B. Pezent, R. Sood, A. Heaton, Near Earth Asteroid (NEA) Scout Solar Sail Contingency Trajectory Design and Analysis, in: Space Flight Mechanics Meeting, American Institute of Aeronautics and Astronautics, Kissimmee, FL, USA, 2018. doi:doi:10.2514/6.2018-0199.

[10] S. Seetha, S. Megala, Aditya-L1 Mission, Current Science. 113 (2017) 610-612.

[11] M.A. Greenhouse, J.C. Mather, M. Clampin, R. Doyon, K.A. Flanagan, M. Franx, H.B. Hammel, J.B. Hutchings, P. Jakobsen, S.J. Lilly, J.I. Lunine, M.J. McCaughrean, M. Mountain, G.H. Rieke, M.J. Rieke, G. Sonneborn, M. Stiavelli, R. Windhorst, G.S. Wright, The James Webb Space Telescope: Mission Overview and Status, in: 2nd International Conference on Space Technology, ICST 2011, 2011. doi:10.1109/ICSpT.2011.6064655.

[12] NASA, Wide-Field InfrarRed Survey Telescope-Astrophysics Focused Telescope Assets WFIRST-AFTA 2015 Report, 2015. http://arxiv.org/abs/1503.03757.

[13] ESA, ARIEL - Assessment Study Report, 2017.

[14] ESA, PLATO - Definition Study Report, 2017. http://sci.esa.int/jump.cfm?oid=59252.

[15] ESA, EUCLID - Assessment Study Report, 2009.

[16] R. Bauske, Operational Maneuver Optimization for the ESA Missions Herschel and Planck, in: Proceeding of 21st International Symposium on Space Flight Dynamics, Toulouse, France, 2009. http://issfd.org/ISSFD_2009/InterMissionDesignI/Bauske.pdf.

[17] Y. Gao, Near-Earth Asteroid Flyby Trajectories from the Sun-Earth L2 for Chang'e-2's Extended Flight, Acta Mechanica Sinica. 29 (2013) 123-131. doi:10.1007/s10409-013-0011-8.

[18] P. Machuca, J.P. Sánchez, S. Greenland, Asteroid flyby opportunities using semi-autonomous CubeSats: Mission design and science opportunities, Planetary and Space Science. (2018). doi:10.1016/j.pss.2018.11.002.

[19] D.L. Richardson, Halo Orbit Formulation for the ISEE-3 Mission, Journal of Guidance, Control, and Dynamics. 3 (1980) 543-548. doi:10.2514/3.56033.

[20] M. Lo, B. Williams, W. Bollman, D. Han, Y. Hahn, J. Bell, E. Hirst, R. Corwin, P. Hong, K. Howell, B. Barden, R. Wilson, M.W. Lo, B. Williams, W.E. Bollman, D. Han, Y. Hahn, J.L. Bell, E.A. Hirst, R.A. Corwin, P.E. Hong, K.C. Howell, B. Barden, R. Wilson, Genesis Mission Design Pasadena , California Specialist Conference, in: AIAA/AAS Astrodynamics Specialist Conference, American Institute of Aeronautics and Astronautics, Boston, MA, USA, 1998.

[21] P. McNamara, G. Racca, Introduction To LISA Pathfinder, 2009.

[22] V. Domingo, B. Fleck, A.I. Poland, The SOHO Mission: An Overview, Solar Physics. (1995). doi:10.1007/BF00733425.

[23] E.C. Stone, A.M. Frandsen, R.A. Mewaldt, E.R. Christian, D. Margolies, J.F. Ormes, F. Snow, The Advanced Composition Explorer, Space Science Reviews. 86 (1998) 1-22. doi:10.1007/978-94-011-4762-0_1.

[24] A. Szabo, NASA Wind Satellite (1994) BT - Handbook of Cosmic Hazards and Planetary Defense, in: J.N. Pelton, F. Allahdadi (Eds.), Springer International Publishing, Cham, 2015: pp. 141-157. doi:10.1007/978-3-31903952-7_13.

[25] J. Burt, B. Smith, Deep Space Climate Observatory: The DSCOVR mission, in: IEEE Aerospace Conference, Institute of Electrical and Electronics Engineers Inc., Big Sky, MT, USA, 2012: pp. 1-13. doi:10.1109/AERO.2012.6187025.

[26] C.L. Bennett, M. Halpern, G. Hinshaw, N. Jarosik, A. Kogut, M. Limon, S.S. Meyer, L. Page, D.N. Spergel, G.S. Tucker, E. Wollack, E.L. Wright, C. Barnes, M.R. Greason, R.S. Hill, E. Komatsu, M.R. Nolta, N. Odegard, H. 
V. Peirs, L. Verde, J.L. Weiland, First Year Wilkinson Microwave Anisotropy Probe (WMAP) Observations: Preliminary Maps and Basic Results, (2003). doi:10.1086/377253.

[27] G.L. Pilbratt, The Herschel Mission, Scientific Objectives, and this Meeting, 2001.

[28] J.A. Tauber, The Planck Mission, Advances in Space Research. (2004). doi:10.1016/j.asr.2003.05.025.

[29] T. Prusti, J.H.J. de Bruijne, A.G.A. Brown, A. Vallenari, The Gaia Mission, Astronomy and Astrophysics. (2016). doi:10.1051/0004-6361/201629272.

[30] R. Bauske, Operational Maneuver Optimization for the ESA Missions Herschel and Planck, in: Proceeding of 21st International Symposium on Space Flight Dynamics, Toulouse, France, 2009.

[31] K. Oshima, S. Campagnola, C.H. Yam, Y. Kayama, Y. Kawakatsu, N. Ozaki, Q. Verspieren, K. Kakihara, K. Oguri, R. Funase, EQUULEUS Mission Analysis: Design of the Transfer Phase, in: International Symposium on Space Technology and Space, Matsuyama, Japan, 2017. https://www.researchgate.net/publication/320143846.

[32] L. Mcnutt, L. Johnson, D. Clardy, Near-Earth Asteroid Scout, in: AIAA SPACE 2014 Conference and Exposition, 2014: pp. 1-9. doi:10.2514/6.2014-4435.

[33] A. Marinan, J. Castillo-Rogez, L. Johnson, J. Dervan, C. Seybold, E. Betts, Near Earth Asteroid (NEA) Scout CubeSat Mission, in: 12th Low-Cost Planetary Missions Conference, Pasadena, CA, 2017.

[34] K. Lemmer, Propulsion for CubeSats, Acta Astronautica. $134 \quad$ (2017) 231-243. doi:10.1016/j.actaastro.2017.01.048.

[35] CU Aerospace, Propulsion Unit for CubeSats, Champaign, IL, USA, 2017.

[36] Busek, BET-100uN Busek Electrospray Thrust, Natick, MA, USA, 2016.

[37] J. Desmars, D. Bancelin, D. Hestroffer, W. Thuillot, J. Desmars, D. Bancelin, D. Hestroffer, W.T. Statistical, J. Desmars, D. Bancelin, D. Hestro, W. Thuillot, Statistical and Numerical Study of Asteroid Orbital Uncertainty, Astronomy and Astrophysics. A32 (2013).

[38] NASA, JPL Center for Near Earth Object Studies, (2008). https://cneos.jpl.nasa.gov/ (accessed January 1, 2018).

[39] NASA, JPL HORIZONS, (2013). http://ssd.jpl.nasa.gov/?horizons (accessed January 1, 2018).

[40] C.L. Goudas, Three-dimensional Periodic Orbits and their Stability, Icarus. 2 (1963) 1-18. https://www.scopus.com/inward/record.uri?eid=2-s2.00001790550\&partnerID $=40 \& m d 5=70548 d 2 e 5 e 610 \mathrm{cdb} 15 \mathrm{f} 70449 \mathrm{a} 03 \mathrm{~d} 0 \mathrm{c} 20$.

[41] K. Deb, A. Pratap, S. Agarwal, T. Meyarivan, A Fast and Elitist Multiobjective Genetic Algorithm: NSGA-II, IEEE Transactions on Evolutionary Computation. 6 (2002) 182-197. doi:10.1109/4235.996017.

[42] G. Gómez, J.J. Masdemont, C. Simó, Quasihalo Orbits Associated with Libration Points, Journal of the Astronautical Sciences. 46 (1998) 135-176. doi:10.1111/cobi.12072.

[43] J.A. Sims, S.N. Flanagan, Preliminary Design of Low-Thrust Missions Interplanetary, in: AIAA/AAS Astrodynamics Specialist Conference, Girdwood, Alaska, 1999.

[44] A. V Rao, D. Benson, G.T. Huntington, C. Francolin, C.L. Darby, M. Patterson, User's Manual for GPOPS Version 2.1: A MATLAB Package for Dynamic Optimization Using the Gauss Pseudospectral Method, Gainesville, FL, USA, 2009.

[45] Y. Kawajir, F. Margot, L. Carl, A. Wächter, Introduction to IPOPT : A tutorial for downloading, installing, and using IPOPT., Pittsburgh, PA, USA, 2011.

[46] G. Gómez, A. Jorba, C. Simó, A. Masdemont, Study of the Transfer Between Halo Orbits, Acta Astronautica. 43 (1998) 493-520.

[47] M. Shirobokov, S.P. Trofimov, M. Ovchinnikov, Survey of Station-Keeping Techniques for Libration Point Orbits Surve, (2017). doi:10.2514/1.G001850. 
[48] R. Walker, D. Koschny, C. Bramanti, E.S.A. Cdf, Miniaturised Asteroid Remote Geophysical Observer (MARGO): A Stand-alone Deep Space CubeSat System for Low- cost Science and Exploration Missions, in: 6th Interplanetary CubeSat Workshop, Cambridge, UK, 2017.

[49] V. Franzese, P. Di Lizia, F. Topputo, Autonomous Optical Navigation for the Lunar Meteoroid Impacts Observer, Journal of Guidance, Control, and Dynamics. (2019) 1-8. doi:10.2514/1.g003999.

[50] P. Machuca, J.P. Sanchez, Autonomous Navigation and Guidance for CubeSats to Flyby Near-Earth Asteroids, in: International Astronautical Congress, Washington, D.C., USA, 2019.

[51] W.S. Koon, M.W. Lo, J.E. Marsden, S.D. Ross, Heteroclinic connections between periodic orbits and resonance transitions in celestial mechanics, Chaos: An Interdisciplinary Journal of Nonlinear Science. 10 (2000) 427-469. doi:10.1063/1.166509.

[52] R.L. Jones, M. Jurić, Ž. Ivezić, Asteroid Discovery and Characterization with the Large Synoptic Survey Telescope, Proceedings of the International Astronomical Union. 10 (2015) 282-292. doi:DOI: $10.1017 /$ S 1743921315008510.

\section{Appendix 1: Catalogue of impulsive asteroid flyby opportunities in CR3BP and ephemeris models}

Table 6

Impulsive asteroid flyby opportunities from Sun-EMB L1 and L2 (CR3BP model).

\begin{tabular}{|c|c|c|c|c|c|c|c|}
\hline Name & From & $\begin{array}{l}\text { Date of Earth } \\
\text { encounter }\end{array}$ & $\begin{array}{l}\text { Absolute } \\
\text { magnitude }\end{array}$ & $\begin{array}{l}\text { Total } \Delta \mathbf{V} \\
(\mathbf{m} / \mathbf{s})\end{array}$ & TOF (days) & $\begin{array}{l}\text { Flyby velocity } \\
(\mathbf{k m} / \mathbf{s})\end{array}$ & $\begin{array}{l}\text { \# of halo } \\
\text { points }^{\mathrm{a}}\end{array}$ \\
\hline $2010 \mathrm{JG}$ & & 2019-Nov-12 & 20.9 & $58.5 \leftrightarrow 74.0$ & $144.1 \leftrightarrow 150.0$ & 16.2 & 2 \\
\hline 2001 FO32 & & 2021-Mar-21 & 17.7 & $31.2 \leftrightarrow 75.8$ & $78.1 \leftrightarrow 150.0$ & 34.2 & 6 \\
\hline 2008 GO20 & & 2021-Jul-27 & 22.3 & $15.9 \leftrightarrow 66.2$ & $97.7 \leftrightarrow 150.0$ & 8.9 & 5 \\
\hline 2016 AJ193 & & 2021-Aug-21 & 18.7 & $25.5 \leftrightarrow 75.5$ & $115.1 \leftrightarrow 150.0$ & 24.7 & 6 \\
\hline 2008 AG33 & & 2022-Apr-28 & 19.6 & $16.9 \leftrightarrow 74.1$ & $98.2 \leftrightarrow 150.0$ & 10.4 & 6 \\
\hline 2006 YT13 & & 2022-Jul-19 & 18.3 & $57.6 \leftrightarrow 61.7$ & $140.9 \leftrightarrow 150.0$ & 23.8 & 2 \\
\hline 2014 HK129 & & 2022-Dec-20 & 21.1 & 68.7 & 150 & 10.8 & 1 \\
\hline 2010 XC15 & & 2022-Dec-27 & 21.4 & $11.6 \leftrightarrow 65.3$ & $141.3 \leftrightarrow 150.0$ & 10.3 & 2 \\
\hline 2015 DG200 & & 2023-Jan-19 & 21.7 & 62.4 & 138.8 & 20.6 & 1 \\
\hline 2012 KY3 & L1 & 2023-Apr-13 & 18.4 & $32.8 \leftrightarrow 64.4$ & $137.5 \leftrightarrow 150.0$ & 19.2 & 3 \\
\hline $2011 \mathrm{GA}$ & & 2023-Oct-15 & 20.8 & $24.5 \leftrightarrow 53.2$ & $99.3 \leftrightarrow 150.0$ & 17.5 & 7 \\
\hline 2018 CC14 & & 2024-Apr-04 & 22.5 & $61.8 \leftrightarrow 64.1$ & $137.8 \leftrightarrow 141.7$ & 9.1 & 2 \\
\hline 2007 ML24 & & 2026-Jul-04 & 19.3 & $25.7 \leftrightarrow 70.8$ & $82.7 \leftrightarrow 150.0$ & 16.8 & 7 \\
\hline 2018 CC14 & & 2027-Apr-07 & 22.5 & $38.4 \leftrightarrow 65.2$ & $126.0 \leftrightarrow 150.0$ & 9.2 & 3 \\
\hline 1999 AN10 & & 2027-Aug-07 & 17.9 & $25.2 \leftrightarrow 51.7$ & $128.6 \leftrightarrow 150.0$ & 26.3 & 3 \\
\hline 2001 WN5 & & 2028-Jun-26 & 18.2 & $0.8 \leftrightarrow 49.8$ & $13.3 \leftrightarrow 150.0$ & 9.9 & 9 \\
\hline 2011 LJ19 & & 2028-Jul-25 & 21.4 & $15.3 \leftrightarrow 48.0$ & $35.5 \leftrightarrow 150.0$ & 9.7 & 8 \\
\hline 1997 XF11 & & 2028-Oct-26 & 16.9 & $1.5 \leftrightarrow 41.7$ & $15.5 \leftrightarrow 114.0$ & 14.2 & 8 \\
\hline 2009 BL71 & & 2029-Apr-01 & 22.1 & $12.1 \leftrightarrow 66.8$ & $110.8 \leftrightarrow 150.0$ & 8.2 & 6 \\
\hline
\end{tabular}




\begin{tabular}{lllllll}
2004 MN4 (Apophis) & 2029-Apr-13 & 19.7 & $15.8 \leftrightarrow 53.8$ & $33.3 \leftrightarrow 145.7$ & 5.6 & 7 \\
\hline 2010 PK9 & 2019-Jul-26 & 21.8 & $43.3 \leftrightarrow 63.6$ & $136.7 \leftrightarrow 150.0$ & 15 & 2 \\
2015 JD1 & 2019-Nov-03 & 20.6 & $21.5 \leftrightarrow 71.2$ & $121.6 \leftrightarrow 141.4$ & 11.5 & 4 \\
2005 WD & 2019-Nov-11 & 21.9 & $60.9 \leftrightarrow 74.1$ & $141.7 \leftrightarrow 150.0$ & 16.8 & 2 \\
2015 BK509 & 2020-Feb-28 & 22.4 & 70.4 & 150 & 14.5 & 1 \\
2009 XO & 2020-May-07 & 20.5 & $13.6 \leftrightarrow 64.8$ & $100.0 \leftrightarrow 150.0$ & 11.8 & 6 \\
2008 TZ3 & 2020-May-10 & 20.4 & $21.5 \leftrightarrow 74.4$ & $109.8 \leftrightarrow 150.0$ & 9.7 & 5 \\
2004 UE & 2021-Nov-13 & 21 & $53.3 \leftrightarrow 63.2$ & $137.4 \leftrightarrow 150.0$ & 14.5 & 2 \\
2017 XC62 & 2022-Jan-24 & 22.4 & $31.7 \leftrightarrow 59.4$ & $141.8 \leftrightarrow 150.0$ & 5 & 2 \\
1989 JA & 2022-May-27 & 17 & $32.9 \leftrightarrow 67.6$ & $118.8 \leftrightarrow 142.2$ & 14 & 3 \\
1998 HH49 & 2023-Oct-17 & 21.3 & $2.1 \leftrightarrow 53.1$ & $53.7 \leftrightarrow 150.0$ & 14.4 & 6 \\
1998 ST27 & 2024-Oct-12 & 19.5 & $11.6 \leftrightarrow 54.9$ & $109.2 \leftrightarrow 150.0$ & 17.4 & 6 \\
2008 DG5 & 2025-Jun-05 & 19.5 & 54.8 & 138.2 & 7.7 & 1 \\
1997 NC1 & 2026-Jun-27 & 17.9 & $5.3 \leftrightarrow 69.8$ & $76.7 \leftrightarrow 150.0$ & 8.7 & 9 \\
1999 AN10 & 2027-Aug-07 & 17.9 & $5.3 \leftrightarrow 40.4$ & $107.2 \leftrightarrow 147.5$ & 26.7 & 4 \\
2012 EY11 & 2027-Sep-19 & 21.7 & $51.5 \leftrightarrow 66.2$ & $150.0 \leftrightarrow 150.0$ & 6.2 & 2 \\
2001 SQ3 & 2028-Mar-19 & 21.6 & $46.9 \leftrightarrow 77.5$ & $122.2 \leftrightarrow 150.0$ & 15.1 & 4 \\
2001 WN5 & 2028-Jun-26 & 18.2 & $16.1 \leftrightarrow 63.5$ & $37.1 \leftrightarrow 150.0$ & 10.1 & 9 \\
2011 LJ19 & 2028-Jul-25 & 21.4 & 58.5 & 150 & 9.9 & 1 \\
2004 OB & 2028-Sep-30 & 18.8 & $41.8 \leftrightarrow 55.3$ & $127.5 \leftrightarrow 150.0$ & 8.6 & 2 \\
2001 XP31 & 2028-Nov-18 & 22.2 & $44.8 \leftrightarrow 78.3$ & $143.8 \leftrightarrow 150.0$ & 11.5 & 3 \\
2004 MN4 (Apophis) & 2029-Apr-13 & 19.7 & $5.5 \leftrightarrow 62.8$ & $55.8 \leftrightarrow 150.0$ & 5.7 & 6 \\
\hline 20.7 &
\end{tabular}

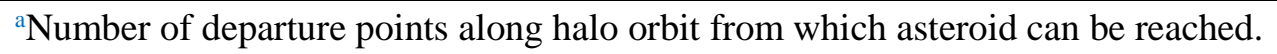

Table 7

Impulsive asteroid flyby opportunities from Sun-EMB L1 and L2 (ephemeris model).

\begin{tabular}{|c|c|c|c|c|c|c|c|}
\hline Name & From & $\begin{array}{l}\text { Date of Earth } \\
\text { encounter }\end{array}$ & $\begin{array}{l}\text { Absolute } \\
\text { magnitude }\end{array}$ & $\begin{array}{l}\text { Total } \Delta \mathbf{V} \\
(\mathbf{m} / \mathbf{s})\end{array}$ & TOF (days) & $\begin{array}{l}\text { Flyby velocity } \\
(\mathbf{k m} / \mathbf{s})\end{array}$ & $\begin{array}{l}\text { \# of halo } \\
\text { points }^{\mathrm{a}}\end{array}$ \\
\hline $2010 \mathrm{JG}$ & & 2019-Nov-12 & 20.9 & $57.5 \leftrightarrow 64.8$ & $144.1 \leftrightarrow 150.0$ & 16.1 & 2 \\
\hline 2001 FO32 & & 2021-Mar-21 & 17.7 & $30.5 \leftrightarrow 62.8$ & $78.1 \leftrightarrow 150.0$ & 34.1 & 5 \\
\hline 2008 GO20 & & 2021-Jul-27 & 22.3 & $14.6 \leftrightarrow 79.7$ & $97.8 \leftrightarrow 150.0$ & 9.1 & 5 \\
\hline 2016 AJ193 & & 2021-Aug-21 & 18.7 & $42.4 \leftrightarrow 64.7$ & $120.9 \leftrightarrow 150.0$ & 21.8 & 4 \\
\hline 2008 AG33 & $\mathrm{L} 1$ & 2022-Apr-28 & 19.6 & $16.9 \leftrightarrow 60.9$ & $98.3 \leftrightarrow 150.1$ & 10.5 & 6 \\
\hline 2006 YT13 & & 2022-Jul-19 & 18.3 & $38.9 \leftrightarrow 59.3$ & $141.0 \leftrightarrow 150.0$ & 15.1 & 2 \\
\hline 2014 HK129 & & 2022-Dec-20 & 21.1 & 47.3 & 150 & 12.8 & 1 \\
\hline 2010 XC15 & & 2022-Dec-27 & 21.4 & $26.7 \leftrightarrow 78.1$ & $141.2 \leftrightarrow 150.0$ & 10.4 & 2 \\
\hline 2015 DG200 & & 2023-Jan-19 & 21.7 & 70.4 & 138.9 & 12.1 & 1 \\
\hline
\end{tabular}




\begin{tabular}{|c|c|c|c|c|c|c|}
\hline 2012 KY3 & 2023-Apr-13 & 18.4 & $34.3 \leftrightarrow 55.2$ & $137.6 \leftrightarrow 150.0$ & 14.9 & 3 \\
\hline $2011 \mathrm{GA}$ & 2023-Oct-15 & 20.8 & $20.0 \leftrightarrow 68.6$ & $99.3 \leftrightarrow 150.0$ & 17.5 & 7 \\
\hline $2018 \mathrm{CC} 14$ & 2024-Apr-04 & 22.5 & 68.5 & 141.6 & 16.2 & 1 \\
\hline 2007 ML24 & 2026-Jul-04 & 19.3 & $29.9 \leftrightarrow 69.7$ & $82.7 \leftrightarrow 150.0$ & 17 & 7 \\
\hline $2018 \mathrm{CC} 14$ & 2027-Apr-07 & 22.5 & $38.3 \leftrightarrow 55.7$ & $126.0 \leftrightarrow 150.1$ & 13.7 & 3 \\
\hline 1999 AN10 & 2027-Aug-07 & 17.9 & $36.2 \leftrightarrow 39.3$ & $128.6 \leftrightarrow 140.2$ & 18.7 & 2 \\
\hline 2001 WN5 & 2028-Jun-26 & 18.2 & $4.4 \leftrightarrow 40.3$ & $13.2 \leftrightarrow 150.4$ & 10.1 & 9 \\
\hline 2011 LJ19 & 2028-Jul-25 & 21.4 & $15.9 \leftrightarrow 54.0$ & $35.4 \leftrightarrow 150.1$ & 10 & 8 \\
\hline 1997 XF11 & 2028-Oct-26 & 16.9 & $2.8 \leftrightarrow 43.1$ & $15.5 \leftrightarrow 113.9$ & 13.6 & 8 \\
\hline 2009 BL71 & 2029-Apr-01 & 22.1 & $20.3 \leftrightarrow 50.3$ & $110.8 \leftrightarrow 150.1$ & 9.7 & 6 \\
\hline 2004 MN4 (Apophis) & 2029-Apr-13 & 19.7 & $20.9 \leftrightarrow 50.7$ & $33.3 \leftrightarrow 145.2$ & 7.2 & 7 \\
\hline 2010 PK9 & 2019-Jul-26 & 21.8 & $43.3 \leftrightarrow 63.6$ & $136.7 \leftrightarrow 150.0$ & 15 & 2 \\
\hline 2015 JD1 & 2019-Nov-03 & 20.6 & $21.5 \leftrightarrow 71.2$ & $121.6 \leftrightarrow 141.4$ & 11.5 & 4 \\
\hline 2005 WD & 2019-Nov-11 & 21.9 & $60.9 \leftrightarrow 74.1$ & $141.7 \leftrightarrow 150.0$ & 16.8 & 2 \\
\hline 2015 ВK509 & 2020-Feb-28 & 22.4 & 70.4 & 150 & 14.5 & 1 \\
\hline $2009 \mathrm{XO}$ & 2020-May-07 & 20.5 & $13.6 \leftrightarrow 64.8$ & $100.0 \leftrightarrow 150.0$ & 11.8 & 6 \\
\hline $2008 \mathrm{TZ3}$ & 2020-May-10 & 20.4 & $21.5 \leftrightarrow 74.4$ & $109.8 \leftrightarrow 150.0$ & 9.7 & 5 \\
\hline $2004 \mathrm{UE}$ & 2021-Nov-13 & 21 & $53.3 \leftrightarrow 63.2$ & $137.4 \leftrightarrow 150.0$ & 14.5 & 2 \\
\hline 2017 XC62 & 2022-Jan-24 & 22.4 & $31.7 \leftrightarrow 59.4$ & $141.8 \leftrightarrow 150.0$ & 5 & 2 \\
\hline $1989 \mathrm{JA}$ & 2022-May-27 & 17 & $32.9 \leftrightarrow 67.6$ & $118.8 \leftrightarrow 142.2$ & 14 & 3 \\
\hline 1998 HH49 & 2023-Oct-17 & 21.3 & $2.1 \leftrightarrow 53.1$ & $53.7 \leftrightarrow 150.0$ & 14.4 & 6 \\
\hline 1998 ST27 & 2024-Oct-12 & 19.5 & $11.6 \leftrightarrow 54.9$ & $109.2 \leftrightarrow 150.0$ & 17.4 & 6 \\
\hline 2008 DG5 & 2025-Jun-05 & 19.5 & 54.8 & 138.2 & 7.7 & 1 \\
\hline $1997 \mathrm{NC} 1$ & 2026-Jun-27 & 17.9 & $5.3 \leftrightarrow 69.8$ & $76.7 \leftrightarrow 150.0$ & 8.7 & 9 \\
\hline 1999 AN10 & 2027-Aug-07 & 17.9 & $5.3 \leftrightarrow 40.4$ & $107.2 \leftrightarrow 147.5$ & 26.7 & 4 \\
\hline 2012 EY11 & 2027-Sep-19 & 21.7 & $51.5 \leftrightarrow 66.2$ & $150.0 \leftrightarrow 150.0$ & 6.2 & 2 \\
\hline 2001 SQ3 & 2028-Mar-19 & 21.6 & $46.9 \leftrightarrow 77.5$ & $122.2 \leftrightarrow 150.0$ & 15.1 & 4 \\
\hline 2001 WN5 & 2028-Jun-26 & 18.2 & $16.1 \leftrightarrow 63.5$ & $37.1 \leftrightarrow 150.0$ & 10.1 & 9 \\
\hline 2011 LJ19 & 2028-Jul-25 & 21.4 & 58.5 & 150 & 9.9 & 1 \\
\hline $2004 \mathrm{OB}$ & 2028-Sep-30 & 18.8 & $41.8 \leftrightarrow 55.3$ & $127.5 \leftrightarrow 150.0$ & 8.6 & 2 \\
\hline 2001 XP31 & 2028-Nov-18 & 22.2 & $44.8 \leftrightarrow 78.3$ & $143.8 \leftrightarrow 150.0$ & 11.5 & 3 \\
\hline 2004 MN4 (Apophis) & 2029-Apr-13 & 19.7 & $5.5 \leftrightarrow 62.8$ & $55.8 \leftrightarrow 150.0$ & 5.7 & 6 \\
\hline
\end{tabular}

${ }^{\mathrm{a}}$ Number of departure points along halo orbit from which asteroid can be reached.

\section{Appendix 2: Multiple-impulse linear optimization problem}

The methodology presented in Section 4.2 is employed to transition from an impulsive maneuver model to a more realistic thrusting model, referred to as quasi-impulsive model. This transition is performed leveraging yet another thrusting model, referred to as intermediate model, in which each maneuver in the original impulsive trajectory is modeled as 
multiple impulses instead. In order to determine the direction of these smaller impulses, the solution to a linearized trajectory optimization problem is employed and presented in the current section.

The optimization problem consists in reaching the final state along a reference trajectory from an initial state along a nominal, uncontrolled trajectory, through multiple impulses (controls) executed at times $t_{i}($ for $i=0, \ldots, N)$. The nominal trajectory of the spacecraft has final state $\boldsymbol{x}_{f}$, and thus the objective of the controls is to reduce the difference $\Delta \boldsymbol{x}_{f}=$ $\boldsymbol{x}_{f}-\boldsymbol{x}_{r e f}\left(t_{f}\right)$. A graphical representation of the optimization problem is provided in Fig. 13.

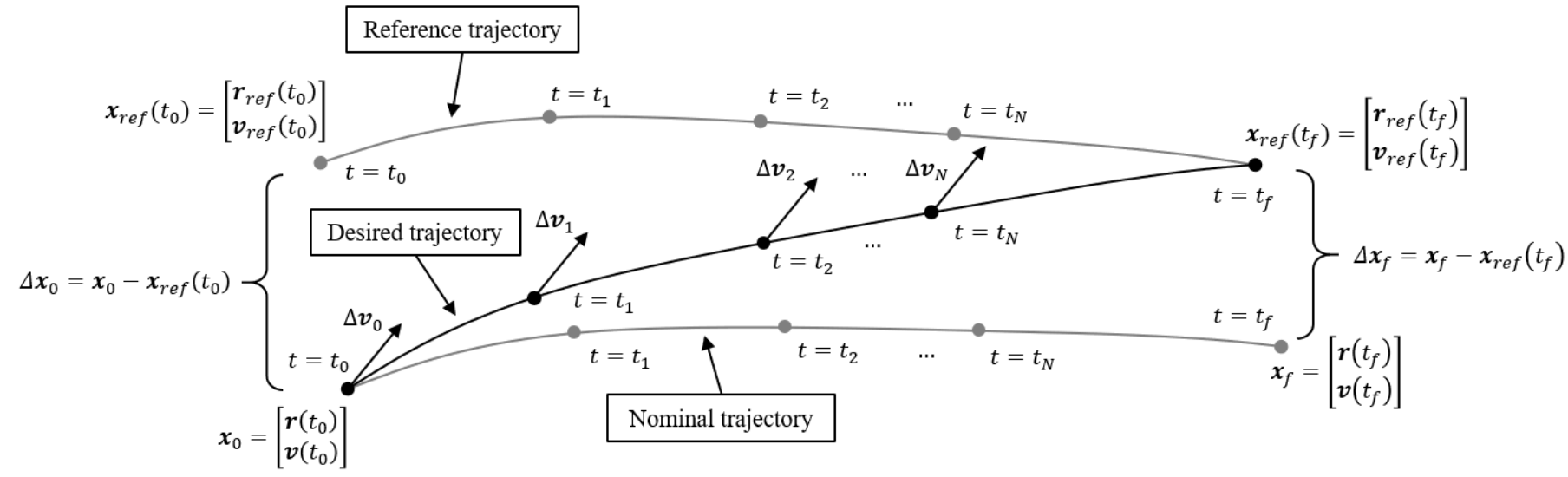

Fig. 13. Multiple-impulse trajectory optimization problem.

The objective function is defined in Eq. (10) as the summation of $\Delta \mathrm{V}$ magnitudes (each of them with associated weighting coefficient $k_{i}$ ), under the final constraint $\Delta \boldsymbol{x}_{f}=\mathbf{0}$.

$$
J=\sum_{i=0}^{N} k_{i} \cdot \Delta \boldsymbol{v}_{i}^{T} \cdot \Delta \boldsymbol{v}_{i}-2 \cdot \lambda^{T} \cdot \Delta \boldsymbol{x}_{f}
$$

where the weighting coefficients may serve as a tool to control the relative magnitude of the impulses. A factor of 2 is included in Eq. (10) multiplying the final constraint for convenience in the development of the equations. Effectively, this factor is integrated in the definition of the weighting coefficients.

The equations of motion along the reference trajectory (with initial state $\boldsymbol{x}_{r e f}\left(t_{0}\right)$ ) are assumed to be linear and equal to those along the nominal trajectory. The state-transition matrix, referred to here as $\underline{\phi}_{i f}$, is used to relate variations in the states at time $t_{i}$ to the variation in the states at time $t_{f}: \underline{\phi}_{i f}=\underline{\boldsymbol{\phi}}\left(t_{i}, t_{f}\right)=\left[\underline{\boldsymbol{\phi}}_{x\left(t_{f}\right) / \boldsymbol{r}\left(t_{i}\right)}, \underline{\boldsymbol{\phi}}_{x\left(t_{f}\right) / \boldsymbol{v}\left(t_{i}\right)}\right]$.

The difference in the final state can therefore be expressed in terms of the initial deviation from the reference trajectory, $\Delta \boldsymbol{x}_{0}=\boldsymbol{x}_{0}-\boldsymbol{x}_{r e f}\left(t_{0}\right)$, the impulses $\Delta \boldsymbol{v}_{i}$, and the state-transition matrices $\underline{\phi}_{i f}$, as in Eq. (11).

$$
\Delta \boldsymbol{x}_{f}=\underline{\boldsymbol{\phi}}_{0 f} \cdot \Delta \boldsymbol{x}_{0}+\underline{\boldsymbol{\phi}}_{0 f} \cdot \Delta \boldsymbol{v}_{0}+\underline{\boldsymbol{\phi}}_{1 f} \cdot \Delta \boldsymbol{v}_{1}+\underline{\boldsymbol{\phi}}_{2 f} \cdot \Delta \boldsymbol{v}_{2}+\cdots+\underline{\boldsymbol{\phi}}_{N f} \cdot \Delta \boldsymbol{v}_{N}=\underline{\boldsymbol{\phi}}_{0 f} \cdot \Delta \boldsymbol{x}_{0}+\sum_{i=0}^{N} \underline{\boldsymbol{\phi}}_{i f} \cdot \Delta \boldsymbol{v}_{i}
$$

where $\underline{\boldsymbol{\phi}}_{i f}=\underline{\phi}_{x}\left(t_{f}\right) / v\left(t_{i}\right)$.

Combining Eq. (10) and Eq. (11), the objective function can thus be expressed in terms of the controls and initial deviation as in Eq. (12).

$$
J=\sum_{i=0}^{N} k_{i} \cdot \Delta \boldsymbol{v}_{i}^{T} \cdot \Delta \boldsymbol{v}_{i}-2 \cdot \lambda^{T} \cdot\left(\underline{\phi}_{0 f} \cdot \Delta \boldsymbol{x}_{0}+\sum_{i=0}^{N} \underline{\boldsymbol{\phi}}_{i f} \cdot \Delta \boldsymbol{v}_{i}\right)
$$

For optimality, the partial derivatives of the objective function with respect to each control shall be equal to zero, such as in Eq. (13).

$$
\left(\frac{\delta J}{\delta \Delta \boldsymbol{v}_{i}}\right)^{T}=2 \cdot k_{i} \cdot \Delta \boldsymbol{v}_{i}-2 \cdot \underline{\boldsymbol{\phi}}_{i f}^{T} \cdot \lambda=\mathbf{0} \quad \text { for } i=0, \ldots, N
$$

From Eq. (13), each impulse can be expressed in terms of the Lagrange multipliers, associated weighting coefficient, and state-transition matrix as in Eq. (14). 


$$
\Delta \boldsymbol{v}_{i}=\frac{\widehat{\boldsymbol{\phi}}_{i f}^{T} \cdot \lambda}{k_{i}} \quad \text { for } i=0, \ldots, N
$$

Finally, substituting Eq. (14) into the definition of the final constraint $\Delta \boldsymbol{x}_{f}=\mathbf{0}$ yields an equation for the Lagrange multipliers (see Eq. (15) and Eq. (16)).

$$
\begin{gathered}
\Delta \boldsymbol{x}_{f}=\underline{\boldsymbol{\phi}}_{0 f} \cdot \Delta \boldsymbol{x}_{0}+\left(\sum_{i=0}^{N} \frac{\widehat{\boldsymbol{\phi}}_{i f} \cdot \underline{\boldsymbol{\phi}_{i f}^{T}}}{k_{i}}\right) \cdot \lambda=\mathbf{0} \\
\lambda=\left(\sum_{i=0}^{N} \frac{\widehat{\boldsymbol{\phi}}_{i f} \cdot \underline{\boldsymbol{\phi}_{i f}^{T}}}{k_{i}}\right)^{-1} \cdot\left(-\underline{\boldsymbol{\phi}}_{0 f} \cdot \Delta \boldsymbol{x}_{0}\right)
\end{gathered}
$$

The expression in Eq. (16) then fully defines the impulses described in Eq. (14).

The solution to this multiple-impulse linearized optimization problem (with equal weighting coefficients for all impulses) is applied to determine the direction of each 1.5-m/s impulse in Section 4.2. This optimization problem is in fact solved once for each impulse, considering only the remaining impulses after that particular maneuver, and employing only the corresponding $\Delta \boldsymbol{v}_{0}$. If the magnitude of $\Delta \boldsymbol{v}_{0}$ from Eq. (14) is larger than $1.5 \mathrm{~m} / \mathrm{s}$, then $\Delta \boldsymbol{v}_{0}$ is also scaled to have the desired magnitude. Finally, and as mentioned in Section 4.2, it may also be necessary to include one or two additional impulses in some cases in order to reach the desired final state (i.e., to encounter the asteroid). 
2019-11-05

\section{High-fidelity trajectory design to flyby near-Earth asteroids using CubeSats}

Machuca, Pablo

elsevier

Machuca P, Sanchez JP, Masdemont JJ, Gomez G. (2019) High-fidelity trajectory design to

flyby near-Earth asteroids using CubeSats. Acta Astronautica, Volume 167, February 2019, pp. 146-163

https://doi.org/10.1016/j.actaastro.2019.09.041

Downloaded from Cranfield Library Services E-Repository 